\title{
Hermite-Hadamard-type inequalities for the interval-valued approximately $h$-convex functions via generalized fractional integrals
}

\author{
Dafang Zhao ${ }^{1,2}$, Muhammad Aamir Ali ${ }^{3^{*}}$ (D) Artion Kashuri ${ }^{4}$, Hüseyin Budak ${ }^{5}$ and Mehmet Zeki Sarikaya ${ }^{5}$
}

\author{
${ }^{*}$ Correspondence: \\ mahr.muhammad.aamir@gmail.com \\ ${ }^{3}$ Jiangsu Key Laboratory for NSLSCS, \\ School of Mathematical Sciences, \\ Nanjing Normal University, Nanjing, \\ Jiangsu 210023, China \\ Full list of author information is \\ available at the end of the article
}

\begin{abstract}
In this paper, we present a new definition of interval-valued convex functions depending on the given function which is called "interval-valued approximately h-convex functions". We establish some inequalities of Hermite-Hadamard type for a newly defined class of functions by using generalized fractional integrals. Our new inequalities are the extensions of previously obtained results like (D.F. Zhao et al. in J. Inequal. Appl. 2018(1):302, 2018 and H. Budak et al. in Proc. Am. Math. Soc., 2019). We also discussed some special cases from our main results.
\end{abstract}

Keywords: Hermite-Hadamard-type inequalities; Interval-valued functions; Fractional integrals

\section{Introduction}

The inequality discovered by Hermite and Hadamard (see [3], [4, pp. 137]) is one of the best-established inequalities in the theory of convex functions with a geometrical interpretation and many applications. These inequalities state that, if $f: I \rightarrow \mathbb{R}$ is a convex function on the interval $I$ of real numbers and $a, b \in I$ with $a<b$, then

$$
f\left(\frac{a+b}{2}\right) \leq \frac{1}{b-a} \int_{a}^{b} f(x) d x \leq \frac{f(a)+f(b)}{2} .
$$

Both inequalities in (1.1) hold in the reversed direction if $f$ is concave. We note that Hermite-Hadamard inequality may be regarded as a refinement of the concept of convexity and it follows easily from Jensen's inequality. Hermite-Hadamard inequality for convex functions has received renewed attention in recent years and a remarkable variety of refinements and generalizations have been studied; see [5-14] and the references therein.

On the other hand, interval analysis is a particular case of set-valued analysis, which is the study of sets in the spirit of mathematical analysis and general topology. It was introduced as an attempt to handle interval uncertainty that appears in many mathematical or computer models of some deterministic real-world phenomena. An old example of an

(c) The Author(s) 2020. This article is licensed under a Creative Commons Attribution 4.0 International License, which permits use sharing, adaptation, distribution and reproduction in any medium or format, as long as you give appropriate credit to the original author(s) and the source, provide a link to the Creative Commons licence, and indicate if changes were made. The images or other third party material in this article are included in the article's Creative Commons licence, unless indicated otherwise in a credit line to the material. If material is not included in the article's Creative Commons licence and your intended use is not permitted by statutory regulation or exceeds the permitted use, you will need to obtain permission directly from the copyright holder. To view a copy of this licence, visit http://creativecommons.org/licenses/by/4.0/. 
interval enclosure is Archimedes' method which is related to computation of the circumference of a circle. In 1966, the first book related to interval analysis was given by Moore who is known as the first user of intervals in computational mathematics; see [15]. After his book, several scientists started to investigate the theory and application of interval arithmetic. Nowadays, because of its applications, interval analysis is a useful tool in the various area which are interested intensely in uncertain data. You can see applications in computer graphics, experimental and computational physics, error analysis, robotics, and many others.

Moreover, several important inequalities (Hermite-Hadamard, Ostrowski, etc.) have been studied for the interval-valued functions in recent years. In [16, 17], Chalco-Cano et al. obtained Ostrowski type inequalities for interval-valued functions by using the Hukuhara derivative for interval-valued functions. In [18], Román-Flores et al. established Minkowski and Beckenbach's inequalities for interval-valued functions. For the others, see [18-22]. However, inequalities were studied for the more general set-valued maps. For example, in [23], Sadowska gave the Hermite-Hadamard inequality. For other studies, you can see $[24,25]$.

The purpose of this paper is to complete the Riemann-Liouville integrals for intervalvalued functions and to obtain the Hermite-Hadamard inequality via these integrals. Furthermore, Hermite-Hadamard-type inequalities are given using these integrals.

\section{Interval calculus}

A real valued interval $X$ is a bounded, closed subset of $\mathbb{R}$ and is defined by

$$
X=[\underline{X}, \bar{X}]=\{t \in \mathbb{R}: \underline{X} \leq t \leq \bar{X}\}
$$

where $\underline{X}, \bar{X} \in \mathbb{R}$ and $\underline{X} \leq \bar{X}$. The numbers $\underline{X}$ and $\bar{X}$ are called the left and the right endpoints of the interval $X$, respectively. When $\bar{X}=\underline{X}=a$, the interval $X$ is said to be degenerate and we use the form $X=a=[a, a]$. Also, we call $X$ positive if $\underline{X}>0$ or negative if $\bar{X}<0$. The set of all closed intervals of $\mathbb{R}$, and the sets of all closed positive intervals of $\mathbb{R}$ and closed negative intervals of $\mathbb{R}$ is denoted by $\mathbb{R}_{\mathcal{I}}, \mathbb{R}_{\mathcal{I}}^{+}$and $\mathbb{R}_{\mathcal{I}}^{-}$, respectively. The Pompeiu-Hausdorff distance between the intervals $X$ and $Y$ is defined by

$$
d(X, Y)=d([\underline{X}, \bar{X}],[\underline{Y}, \bar{Y}])=\max \{\underline{\mid X}-\underline{Y}|,| \bar{X}-\bar{Y} \mid\} .
$$

It is well known that $\left(\mathbb{R}_{\mathcal{I}}, d\right)$ is a complete metric space; see [26].

Now, we give the definitions of basic interval arithmetic operations for the intervals $X$ and $Y$ as follows:

$$
\begin{aligned}
& X+Y=[\underline{X}+\underline{Y}, \bar{X}+\bar{Y}], \\
& X-Y=[\underline{X}-\bar{Y}, \bar{X}-\underline{Y}], \\
& X \cdot Y=[\min S, \max S] \quad \text { where } S=\{\underline{X} \underline{Y}, \underline{X} \bar{Y}, \bar{X} \underline{Y}, \bar{X} \bar{Y}\}, \\
& X / Y=[\min T, \max T] \quad \text { where } T=\{\underline{X} / \underline{Y}, \underline{X} / \bar{Y}, \bar{X} / \underline{Y}, \bar{X} / \bar{Y}\} \text { and } 0 \notin Y .
\end{aligned}
$$


Scalar multiplication of the interval $X$ is defined by

$$
\lambda X=\lambda[\underline{X}, \bar{X}]= \begin{cases}{[\lambda \underline{X}, \lambda \bar{X}],} & \lambda>0 ; \\ \{0\}, & \lambda=0 ; \\ {[\lambda \bar{X}, \lambda \underline{X}],} & \lambda<0,\end{cases}
$$

where $\lambda \in \mathbb{R}$.

The opposite of the interval $X$ is

$$
-X:=(-1) X=[-\bar{X},-\underline{X}],
$$

where $\lambda=-1$.

The subtraction is given by

$$
X-Y=X+(-Y)=[\underline{X}-\bar{Y}, \bar{X}-\underline{Y}] .
$$

In general, $-X$ is not additive inverse for $X$, i.e. $X-X \neq 0$.

The definitions of operations lead to a number of algebraic properties which allows $\mathbb{R}_{\mathcal{I}}$ to be a quasilinear space; see [27]. They can be listed as follows (see [15, 26-29]):

(1) (Associativity of addition) $(X+Y)+Z=X+(Y+Z)$ for all $X, Y, Z \in \mathbb{R}_{\mathcal{I}}$,

(2) (Additivity element) $X+0=0+X=0$ for all $X \in \mathbb{R}_{\mathcal{I}}$,

(3) (Commutativity of addition) $X+Y=Y+X$ for all $X, Y \in \mathbb{R}_{\mathcal{I}}$,

(4) (Cancelation law) $X+Z=Y+Z \Longrightarrow X=Y$ for all $X, Y, Z \in \mathbb{R}_{\mathcal{I}}$,

(5) (Associativity of multiplication) $(X \cdot Y) \cdot Z=X \cdot(Y \cdot Z)$ for all $X, Y, Z \in \mathbb{R}_{\mathcal{I}}$,

(6) (Commutativity of multiplication) $X \cdot Y=Y \cdot X$ for all $X, Y \in \mathbb{R}_{\mathcal{I}}$,

(7) (Unity element) $X \cdot 1=1 \cdot X$ for all $X \in \mathbb{R}_{\mathcal{I}}$,

(8) (Associativity law) $\lambda(\mu X)=(\lambda \mu) X$ for all $X \in \mathbb{R}_{\mathcal{I}}$ and all $\lambda, \mu \in \mathbb{R}$,

(9) (First distributivity law) $\lambda(X+Y)=\lambda X+\lambda Y$ for all $X, Y \in \mathbb{R}_{\mathcal{I}}$ and all $\lambda \in \mathbb{R}$,

(10) (Second distributivity law) $(\lambda+\mu) X=\lambda X+\mu X$ for all $X \in \mathbb{R}_{\mathcal{I}}$ and all $\lambda, \mu \in \mathbb{R}$.

Besides these properties, the distributive law is not always valid for intervals. For example, $X=[1,2], Y=[2,3]$ and $Z=[-2,-1]$.

$$
X \cdot(Y+Z)=[0,4]
$$

whereas

$$
X \cdot Y+X \cdot Z=[-2,5] .
$$

But this law holds in certain cases. If $Y \cdot Z>0$, then

$$
X \cdot(Y+Z)=X \cdot Y+X \cdot Z \text {. }
$$

Moreover, one of the set properties is the inclusion $\subseteq$ that is given by

$$
X \subseteq Y \quad \Longleftrightarrow \quad \underline{Y} \leq \underline{X} \text { and } \bar{X} \leq \bar{Y} .
$$


Considering together with arithmetic operations and inclusion, one has the following property which is called the inclusion isotone of interval operations:

Let $\odot$ be the addition, multiplication, subtraction or division. If $X, Y, Z$ and $T$ are intervals such that

$$
X \subseteq Y \text { and } Z \subseteq T
$$

then the following relation is valid:

$$
X \odot Z \subseteq Y \odot T .
$$

The following proposition is about scalar multiplication preserving the inclusion.

Proposition 1 Let $X, Y$ be intervals and $\lambda \in \mathbb{R}$. If $X \subseteq Y$, then $\lambda X \subseteq \lambda Y$.

\subsection{Integral of interval-valued functions}

In this section, the notion of integral is mentioned for interval-valued functions. Before the definition of integral, the necessary concepts will be given as follows:

A function $F$ is said to be an interval-valued function of $t$ on $[a, b]$, if it assigns a nonempty interval to each $t \in[a, b]$

$$
F(t)=[\underline{F}(t), \bar{F}(t)] .
$$

A partition of $[a, b]$ is any finite ordered subset $P$ having the form

$$
P: a=t_{0}<t_{1}<\cdots<t_{n}=b .
$$

The mesh of a partition $P$ is defined by

$$
\operatorname{mesh}(P)=\max \left\{t_{i}-t_{i-1}: i=1,2, \ldots, n\right\} .
$$

We denote by $P([a, b])$ the set of all partitions of $[a, b]$. Let $P(\delta,[a, b])$ be the set of all $P \in P([a, b])$ such that mesh $(P)<\delta$. Choose an arbitrary point $\xi_{i}$ in the interval $\left[t_{i-1}, t_{i}\right]$, $(i=1,2, \ldots, n)$ and let us define the sum

$$
S(F, P, \delta)=\sum_{i=1}^{n} F\left(\xi_{i}\right)\left[t_{i}-t_{i-1}\right]
$$

where $F:[a, b] \rightarrow \mathbb{R}_{\mathcal{I}}$. We call $S(F, P, \delta)$ a Riemann sum of $F$ corresponding to $P \in$ $P(\delta,[a, b])$.

Definition 1 ([30-32]) A function $F:[a, b] \rightarrow \mathbb{R}_{\mathcal{I}}$ is called interval Riemann integrable ((IR)-integrable) on $[a, b]$, if there exists $A \in \mathbb{R}_{I}$ such that, for each $\varepsilon>0$, there exists $\delta>0$ such that

$$
d(S(F, P, \delta), A)<\varepsilon
$$


for every Riemann sum $S$ of $F$ corresponding to each $P \in P(\delta,[a, b])$ and independent from choice of $\xi_{i} \in\left[t_{i-1}, t_{i}\right]$ for all $1 \leq i \leq n$. In this case, $A$ is called the (IR)-integral of $F$ on $[a, b]$ and is denoted by

$$
A=(I R) \int_{a}^{b} F(t) d t
$$

The collection of all functions that are (IR)-integrable on $[a, b]$ will be denoted by $\mathcal{I} \mathcal{R}_{([a, b])}$.

The following theorem gives a relation between $(I R)$-integrable and Riemann integrable (R-integrable) (see [33], p. 131):

Theorem 1 Let $F:[a, b] \rightarrow \mathbb{R}_{\mathcal{I}}$ be an interval-valued function such that $F(t)=[\underline{F}(t), \bar{F}(t)]$. $F \in \mathcal{I R}_{([a, b])}$ if and only if $\underline{F}(t), \bar{F}(t) \in \mathcal{R}_{([a, b])}$ and

$$
(I R) \int_{a}^{b} F(t) d t=\left[(R) \int_{a}^{b} \underline{F}(t) d t,(R) \int_{a}^{b} \bar{F}(t) d t\right]
$$

where $\mathcal{R}_{([a, b])}$ denotes the all $R$-integrable functions.

It is seen easily that, if $F(t) \subseteq G(t)$ for all $t \in[a, b]$, then

$$
(I R) \int_{a}^{b} F(t) d t \subseteq(I R) \int_{a}^{b} G(t) d t .
$$

In $[1,34]$, Zhao et al. introduced a kind of convex interval-valued function as follows.

Definition 2 Let $h:[c, d] \rightarrow \mathbb{R}$ be a non-negative function, $(0,1) \subseteq[c, d]$ and $h \neq 0$. We say that $F:[a, b] \rightarrow \mathbb{R}_{\mathcal{I}}^{+}$is a $h$-convex interval-valued function, if for all $x, y \in[a, b]$ and $t \in(0,1)$, we have

$$
h(t) F(x)+h(1-t) F(y) \subseteq F(t x+(1-t) y) .
$$

With $\operatorname{SX}\left(h,[a, b], \mathbb{R}_{\mathcal{I}}^{+}\right)$will show the set of all $h$-convex interval-valued functions.

The usual notion of convex interval-valued function corresponds to relation (2.1) with $h(t)=t$, see [23]. Also, if we take $h(t)=t^{s}$ in (2.1), then Definition 2 gives the other convex interval-valued function defined by Breckner; see [35].

Otherwise, Zhao et al. obtained the following Hermite-Hadamard inequality for interval-valued functions by using $h$-convexity.

Theorem 2 ([1]) Let $F:[a, b] \rightarrow \mathbb{R}_{\mathcal{I}}^{+}$be an interval-valued function such that $F(t)=$ $[\underline{F}(t), \bar{F}(t)]$ and $F \in \mathcal{I R}_{([a, b])}, h:[0,1] \rightarrow \mathbb{R}$ be a non-negative function and $h\left(\frac{1}{2}\right) \neq 0$. If $F \in \mathrm{SX}\left(h,[a, b], \mathbb{R}_{\mathcal{I}}^{+}\right)$, then

$$
\frac{1}{2 h\left(\frac{1}{2}\right)} F\left(\frac{a+b}{2}\right) \supseteq \frac{1}{b-a}(I R) \int_{a}^{b} F(x) d x \supseteq[F(a)+F(b)] \int_{0}^{1} h(t) d t .
$$


Remark 1 (i) If $h(t)=t$, then (2.2) reduces to the following result:

$$
F\left(\frac{a+b}{2}\right) \supseteq \frac{1}{b-a}(I R) \int_{a}^{b} F(x) d x \supseteq \frac{F(a)+F(b)}{2},
$$

which is obtained by Sadowska in [23].

(ii) If $h(t)=t^{s}$, then (2.2) reduces to the following result:

$$
2^{s-1} F\left(\frac{a+b}{2}\right) \supseteq \frac{1}{b-a}(I R) \int_{a}^{b} F(x) d x \supseteq \frac{F(a)+F(b)}{s+1},
$$

which is obtained by Osuna-Gomez et al. in [36].

Theorem 3 Let $F, G:[a, b] \rightarrow \mathbb{R}_{\mathcal{I}}^{+}$be two interval-valued functions such that $F(t)=$ $[\underline{F}(t), \bar{F}(t)]$ and $G(t)=[\underline{G}(t), \bar{G}(t)]$, where $F, G \in \mathcal{I R}_{([a, b])}, h_{1}, h_{2}:[0,1] \rightarrow \mathbb{R}$ are two nonnegative functions and $h_{1}\left(\frac{1}{2}\right) h_{2}\left(\frac{1}{2}\right) \neq 0$. If $F, G \in \mathrm{SX}\left(h,[a, b], \mathbb{R}_{\mathcal{I}}^{+}\right)$, then

$$
\begin{aligned}
& \frac{1}{2 h_{1}\left(\frac{1}{2}\right) h_{2}\left(\frac{1}{2}\right)} F\left(\frac{a+b}{2}\right) G\left(\frac{a+b}{2}\right) \\
& \supseteq \frac{1}{b-a}(I R) \int_{a}^{b} F(x) G(x) d x+M(a, b)(I R) \int_{0}^{1} h_{1}(t) h_{2}(1-t) d t \\
& \quad+N(a, b)(I R) \int_{0}^{1} h_{1}(t) h_{2}(t) d t
\end{aligned}
$$

and

$$
\begin{aligned}
& \frac{1}{b-a}(I R) \int_{a}^{b} F(x) G(x) d x \\
& \quad \supseteq M(a, b)(I R) \int_{0}^{1} h_{1}(t) h_{2}(t) d t+N(a, b)(I R) \int_{0}^{1} h_{1}(t) h_{2}(1-t) d t,
\end{aligned}
$$

where

$$
M(a, b)=F(a) G(a)+F(b) G(b) \quad \text { and } \quad N(a, b)=F(a) G(b)+F(b) G(a) .
$$

Remark 2 If $h(t)=t$, then (2.5) reduces to the following result:

$$
\frac{1}{b-a}(I R) \int_{a}^{b} F(x) G(x) d x \supseteq \frac{1}{3} M(a, b)+\frac{1}{6} N(a, b) .
$$

Remark 3 If $h(t)=t$, then (2.4) reduces to the following result:

$$
2 F\left(\frac{a+b}{2}\right) G\left(\frac{a+b}{2}\right) \supseteq \frac{1}{b-a}(I R) \int_{a}^{b} F(x) G(x) d x+\frac{1}{6} M(a, b)+\frac{1}{3} N(a, b) .
$$

In [2], Budak et al. obtained the following inequalities of Hermite-Hadamard type for the convex interval-valued functions. 
Theorem 4 If $F:[a, b] \rightarrow \mathbb{R}_{\mathcal{I}}^{+}$is a convex interval-valued function such that $F(t)=$ $[\underline{F}(t), \bar{F}(t)]$ and $\alpha>0$, then we have

$$
F\left(\frac{a+b}{2}\right) \supseteq \frac{\Gamma(\alpha+1)}{2(b-a)^{\alpha}}\left[J_{a+}^{\alpha} F(b)+J_{b-}^{\alpha} F(a)\right] \supseteq \frac{F(a)+F(b)}{2} .
$$

Theorem 5 If $F, G:[a, b] \rightarrow \mathbb{R}_{\mathcal{I}}^{+}$are two convex interval-valued functions such that $F(t)=$ $[\underline{F}(t), \bar{F}(t)]$ and $G(t)=[\underline{G}(t), \bar{G}(t)]$, then for $\alpha>0$ we have

$$
\begin{aligned}
& \frac{\Gamma(\alpha+1)}{2(b-a)^{\alpha}}\left[J_{a+}^{\alpha} F(b) G(b)+J_{b-}^{\alpha} F(a) G(a)\right] \\
& \quad \supseteq\left(\frac{1}{2}-\frac{\alpha}{(\alpha+1)(\alpha+2)}\right) M(a, b)+\frac{\alpha}{(\alpha+1)(\alpha+2)} N(a, b)
\end{aligned}
$$

and

$$
\begin{aligned}
& 2 F\left(\frac{a+b}{2}\right) G\left(\frac{a+b}{2}\right) \\
& \supseteq \frac{\Gamma(\alpha+1)}{2(b-a)^{\alpha}}\left[J_{a+}^{\alpha} F(b) G(b)+J_{b-}^{\alpha} F(a) G(a)\right] \\
&+\frac{\alpha}{(\alpha+1)(\alpha+2)} M(a, b)+\left(\frac{1}{2}-\frac{\alpha}{(\alpha+1)(\alpha+2)}\right) N(a, b),
\end{aligned}
$$

where $M(a, b)$ and $N(a, b)$ are defined in Theorem 3.

For the other fractional inequalities for the convex interval-valued functions, see [37].

\section{Interval-valued approximately convexities}

In this section we define a new class of interval-valued approximately $h$-convex functions, which is depending upon a given function. We discuss some special cases of our new definition and find new definitions of approximately $h$-convex functions. We let $\left(X,\|\cdot\|_{I}\right)$ be a normed quasilinear space, let $I$ be a nonempty interval-valued convex subset of $X$, $H: X \times X \rightarrow \mathbb{R}$ and let $h:(0,1) \rightarrow \mathbb{R}$ be the given functions.

Definition 3 A function $F: I \rightarrow \mathbb{R}_{\mathcal{I}}^{+}$is said to be interval-valued approximately $h$-convex function, if

$$
F(t x+(1-t) y) \supseteq h(t) F(x)+h(1-t) F(y)+H(x, y),
$$

for all $t \in(0,1)$ and $a, b \in I$.

Now we discuss some special cases of Definition 3.

I. If we use $H(x, y)=\epsilon(\|x-y\|)^{\gamma}$ for some $\epsilon \in \mathbb{R}$ and $\gamma>1$ in Definition 3, then we have a new definition of an interval-valued approximately convex function which is called an interval-valued $\gamma$-approximately $h$-convex function.

Definition 4 A function $F: I \rightarrow \mathbb{R}_{\mathcal{I}}^{+}$is said to be an interval-valued $\gamma$-approximately $h$ convex function, if

$$
F(t x+(1-t) y) \supseteq h(t) F(x)+h(1-t) F(y)+\epsilon(\|x-y\|)^{\gamma},
$$

for all $t \in(0,1)$ and $a, b \in I$. 
II. If we use $H(x, y)=\epsilon(\|x-y\|)$ for some $\epsilon \in \mathbb{R}$ in Definition 3, then we have a new definition of an interval-valued approximately convex function which is called an interval-valued $\epsilon$-approximately $h$-convex function.

Definition 5 A function $F: I \rightarrow \mathbb{R}_{\mathcal{I}}^{+}$is said to be an interval-valued $\epsilon$-approximately $h$ convex function, if

$$
F(t x+(1-t) y) \supseteq h(t) F(x)+h(1-t) F(y)+\epsilon(\|x-y\|)
$$

for all $t \in(0,1)$ and $a, b \in I$.

III. If we use $H(x, y)=-\mu t(1-t)\|y-x\|^{2}$ for some $\mu>0$ in Definition 3, then we have a new definition of an interval-valued approximately convex function which is called an interval-valued strongly $h$-convex function.

Definition 6 A function $F: I \rightarrow \mathbb{R}_{\mathcal{I}}^{+}$is said to be an interval-valued strongly $h$-convex function, if

$$
F(t x+(1-t) y) \supseteq h(t) F(x)+h(1-t) F(y)-\mu t(1-t)\|y-x\|^{2},
$$

for all $t \in(0,1)$ and $a, b \in I$.

IV. If we use $H(x, y)=\mu t(1-t)\|y-x\|^{2}$ for some $\mu>0$ in Definition 3, then we have a new definition of interval-valued approximately convex function which is called an interval-valued relaxed $h$-convex function.

Definition 7 A function $F: I \rightarrow \mathbb{R}_{\mathcal{I}}^{+}$is said to be an interval-valued relaxed $h$-convex function, if

$$
F(t x+(1-t) y) \supseteq h(t) F(x)+h(1-t) F(y)+\mu t(1-t)\|y-x\|^{2},
$$

for all $t \in(0,1)$ and $a, b \in I$.

V. If we use $\gamma=0$ in Definition 4 or $\epsilon=0$ in Definition 5, we have Definition 2.

\section{Interval-valued generalized fractional integral operators}

In this section, we define a generalized fractional integral operator for the interval-valued functions and discuss special cases of our newly define integral operator.

Let us define a function $\varphi:[0,+\infty) \rightarrow[0,+\infty)$ satisfying the following conditions:

$$
\begin{aligned}
& \int_{0}^{1} \frac{\varphi(t)}{t} d t<+\infty, \\
& \frac{1}{A_{1}} \leq \frac{\varphi(s)}{\varphi(r)} \leq A_{1} \quad \text { for } \frac{1}{2} \leq \frac{s}{r} \leq 2, \\
& \frac{\varphi(r)}{r^{2}} \leq A_{2} \frac{\varphi(s)}{s^{2}} \quad \text { for } s \leq r, \\
& \left|\frac{\varphi(r)}{r^{2}}-\frac{\varphi(s)}{s^{2}}\right| \leq A_{3}|r-s| \frac{\varphi(r)}{r^{2}} \quad \text { for } \frac{1}{2} \leq \frac{s}{r} \leq 2,
\end{aligned}
$$


where $A_{1}, A_{2}, A_{3}>0$ are independent of $r, s>0$. If $\varphi(r) r^{\alpha}$ is increasing for some $\alpha \geq 0$ and $\frac{\varphi(r)}{r^{\beta}}$ is decreasing for some $\beta \geq 0$, then $\varphi$ satisfies (4.1)-(4.4); see [12]. Meanwhile, in [11], Sarikaya and Ertuğral defined the following generalized fractional integrals:

$$
\begin{aligned}
& { }_{a+} I_{\varphi} f(x)=\int_{a}^{x} \frac{\varphi(x-t)}{x-t} f(t) d t, \quad x>a, \\
& { }_{b-} I_{\varphi} f(x)=\int_{x}^{b} \frac{\varphi(t-x)}{t-x} f(t) d t, \quad x<b .
\end{aligned}
$$

Therefore, we can give the following new definitions.

Definition 8 Let $F:[a, b] \rightarrow \mathbb{R}_{\mathcal{I}}$ be an interval-valued function such that $F(t)=[\underline{F}(t), \bar{F}(t)]$ and $F \in \mathcal{I R}_{([a, b])}$. Then the interval-valued left-sided and right-sided generalized fractional integrals of the function $F$, respectively, are given as

$$
\begin{aligned}
& { }_{a+} \Im_{\varphi} F(x)=(I R) \int_{a}^{x} \frac{\varphi(x-t)}{x-t} F(t) d t, \quad x>a, \\
& { }_{b-} \Im_{\varphi} F(x)=(I R) \int_{x}^{b} \frac{\varphi(t-x)}{t-x} F(t) d t, \quad x<b .
\end{aligned}
$$

Corollary 1 Let $F:[a, b] \rightarrow \mathbb{R}_{\mathcal{I}}$ be an interval-valued function such that $F(t)=[\underline{F}(t), \bar{F}(t)]$ and $F \in \mathcal{I R}_{([a, b])}$. Then, we have

$$
{ }_{a+} \mathfrak{I}_{\varphi} F(x)=\left[{ }_{a+} I_{\varphi} \underline{F}(x),{ }_{a+} I_{\varphi} \bar{F}(x)\right]
$$

and

$$
{ }_{b-} \Im_{\varphi} F(x)=\left[{ }_{b-} I_{\varphi} \underline{F}(x),{ }_{b-} I_{\varphi} \bar{F}(x)\right] .
$$

The most important feature of interval-valued generalized fractional integrals is that they generalize some types of fractional integrals such as the Riemann-Liouville fractional integral, the $k$-Riemann-Liouville fractional integral, Katugampola fractional integrals, the conformable fractional integral, and Hadamard fractional integrals. These important special cases of the integral operators (4.5) and (4.6) are mentioned below.

i) Taking $\varphi(t)=t$, the operators (4.5) and (4.6) reduce to the interval-valued Riemann integrals as follows:

$$
\begin{aligned}
& I_{a^{+}} F(x)=(I R) \int_{a}^{x} F(t) d t, \quad x>a, \\
& I_{b^{-}} F(x)=(I R) \int_{x}^{b} F(t) d t, \quad x<b .
\end{aligned}
$$

ii) Taking $\varphi(t)=\frac{t^{\alpha}}{\Gamma(\alpha)}$, the operators (4.5) and (4.6) reduce to the interval-valued Riemann-Liouville fractional integrals as follows:

$$
\begin{aligned}
& J_{a^{+}}^{\alpha} F(x)=\frac{1}{\Gamma(\alpha)}(I R) \int_{a}^{x}(x-t)^{\alpha-1} F(t) d t, \quad x>a, \\
& J_{b^{-}}^{\alpha} F(x)=\frac{1}{\Gamma(\alpha)}(I R) \int_{x}^{b}(t-x)^{\alpha-1} F(t) d t, \quad x<b .
\end{aligned}
$$


iii) Taking $\varphi(t)=\frac{t^{\frac{\alpha}{k}}}{k \Gamma_{k}(\alpha)}$, the operators (4.5) and (4.6) reduce to the interval-valued $k$ Riemann-Liouville fractional integrals as follows:

$$
\begin{aligned}
& I_{a^{+}, k}^{\alpha} F(x)=\frac{1}{k \Gamma_{k}(\alpha)}(I R) \int_{a}^{x}(x-t)^{\frac{\alpha}{k}-1} F(t) d t, \quad x>a, \\
& I_{b^{-}, k}^{\alpha} F(x)=\frac{1}{k \Gamma_{k}(\alpha)}(I R) \int_{x}^{b}(t-x)^{\frac{\alpha}{k}-1} F(t) d t, \quad x<b,
\end{aligned}
$$

where

$$
\Gamma_{k}(\alpha)=\int_{0}^{\infty} t^{\alpha-1} e^{-\frac{t^{k}}{k}} d t, \quad \mathcal{R}(\alpha)>0,
$$

and

$$
\Gamma_{k}(\alpha)=k^{\frac{\alpha}{k}-1} \Gamma\left(\frac{\alpha}{k}\right), \quad \mathcal{R}(\alpha)>0 ; k>0 .
$$

iv) Taking $\varphi(t)=t(x-t)^{\alpha-1}$, the operator (4.5) reduces to the interval-valued conformable fractional operator as follows:

$$
I_{a}^{\alpha} F(x)=(I R) \int_{a}^{x} t^{\alpha-1} F(t) d t=(I R) \int_{a}^{x} F(t) d_{\alpha} t, \quad x>a, \alpha \in(0,1) .
$$

\section{Main results}

In this section, we prove some inequalities of Hermite-Hadamard type for the intervalvalued approximately $h$-convex functions via generalized fractional integrals. We use for brevity the following notations in the next new results:

$$
\Lambda(x)=\int_{0}^{x} \frac{\varphi((b-a) t)}{t} d t<+\infty
$$

and

$$
\psi(x)=\int_{0}^{x} \frac{\varphi\left(\frac{(b-a)}{2} t\right)}{t} d t<+\infty .
$$

Theorem 6 If $F:[a, b] \rightarrow \mathbb{R}_{\mathcal{I}}^{+}$is an interval-valued approximately h-convex function such that $F(t)=[\underline{F}(t), \bar{F}(t)]$, then we have the following inequalities for the generalized fractional integrals:

$$
\begin{aligned}
& \frac{1}{2 h\left(\frac{1}{2}\right)} F\left(\frac{a+b}{2}\right)-\frac{1}{2 h\left(\frac{1}{2}\right) \Lambda(1)} \int_{a}^{b} \frac{\varphi(b-x)}{b-x} H(x, a+b-x) d x \\
& \supseteq \supseteq \frac{1}{2 \Lambda(1)}\left[a+\Im_{\varphi} F(b)+{ }_{b-} \Im_{\varphi} F(a)\right] \\
& \supseteq \supseteq \frac{[F(a)+F(b)]}{2 \Lambda(1)} \int_{0}^{1}[h(t)+h(1-t)] \frac{\varphi((b-a) t)}{t} d t+H(a, b) .
\end{aligned}
$$

Proof Since $F$ is interval-valued approximately $h$-convex function, we have

$$
\frac{1}{h\left(\frac{1}{2}\right)} F\left(\frac{x+y}{2}\right) \supseteq F(x)+F(y)+\frac{1}{h\left(\frac{1}{2}\right)} H(x, y) .
$$


By setting $x=t a+(1-t) b$ and $y=t b+(1-t) a$ in (5.2), we obtain

$$
\begin{aligned}
\frac{1}{h\left(\frac{1}{2}\right)} F\left(\frac{a+b}{2}\right) \supseteq & F(t a+(1-t) b)+F(t b+(1-t) a) \\
& +\frac{1}{h\left(\frac{1}{2}\right)} H(t a+(1-t) b, t b+(1-t) a) .
\end{aligned}
$$

Multiplying both sides of (5.3) by $\frac{\varphi((b-a) t)}{t}$ and integrating the resultant one with respect to $t$ over $[0,1]$, we get

$$
\begin{aligned}
& \frac{1}{h\left(\frac{1}{2}\right)} F\left(\frac{a+b}{2}\right) \int_{0}^{1} \frac{\varphi((b-a) t)}{t} d t \\
& \quad \supseteq\left\{(I R) \int_{0}^{1} \frac{\varphi((b-a) t)}{t} F(t a+(1-t) b) d t+(I R) \int_{0}^{1} \frac{\varphi((b-a) t)}{t} F(t b+(1-t) a) d t\right. \\
& \left.\quad+\frac{1}{h\left(\frac{1}{2}\right)} \int_{0}^{1} \frac{\varphi((b-a) t)}{t} H(t a+(1-t) b, t b+(1-t) a) d t\right\}
\end{aligned}
$$

In Eq. (5.4), using Theorem 1, we obtain

$$
\text { (IR) } \begin{aligned}
& \int_{0}^{1} \frac{\varphi((b-a) t)}{t} F(t a+(1-t) b) d t \\
= & {\left[(R) \int_{0}^{1} \frac{\varphi((b-a) t)}{t} \underline{F}(t a+(1-t) b) d t,(R) \int_{0}^{1} \frac{\varphi((b-a) t)}{t} \bar{F}(t a+(1-t) b) d t\right] } \\
= & {\left[(R) \int_{a}^{b} \frac{\varphi(b-x)}{b-x} \underline{F}(x) d x,(R) \int_{a}^{b} \frac{\varphi(b-x)}{b-x} \bar{F}(x) d x\right] } \\
= & {\left[a_{+} I_{\varphi} \underline{F}(b), a_{a+} I_{\varphi} \bar{F}(b)\right] } \\
= & a_{a+} \mathfrak{I}_{\varphi} F(b) .
\end{aligned}
$$

Similarly, we have

$$
\begin{aligned}
\text { (IR) } & \int_{0}^{1} \frac{\varphi((b-a) t)}{t} F(t b+(1-t) a) d t \\
= & {\left[(R) \int_{0}^{1} \frac{\varphi((b-a) t)}{t} \underline{F}(t b+(1-t) a) d t,(R) \int_{0}^{1} \frac{\varphi((b-a) t)}{t} \bar{F}(t b+(1-t) a) d t\right] } \\
= & { }_{b-} \Im_{\varphi} F(a) .
\end{aligned}
$$

Hence, we achieved our first inequality. To prove the second inequality since $F$ is intervalvalued approximately $h$-convex function, we get

$$
F(t a+(1-t) b) \supseteq h(t) F(a)+h(1-t) F(b)+H(a, b)
$$

and

$$
F(t b+(1-t) a) \supseteq h(t) F(b)+h(1-t) F(a)+H(a, b) .
$$


Adding (5.5) and (5.6), we have

$$
F(t a+(1-t) b)+F(t b+(1-t) a) \supseteq[h(t)+h(1-t)][F(a)+F(b)]+2 H(a, b) .
$$

Multiplying (5.7) by $\frac{\varphi((b-a) t)}{t}$ on both sides and integrating the resultant one with respect to $t$ over $[0,1]$, we have

$$
\begin{aligned}
& \text { (IR) } \int_{0}^{1} \frac{\varphi((b-a) t)}{t} F(t a+(1-t) b) d t+(I R) \int_{0}^{1} \frac{\varphi((b-a) t)}{t} F(t b+(1-t) a) d t \\
& \supseteq[F(a)+F(b)] \int_{0}^{1} \frac{\varphi((b-a) t)}{t}[h(t)+h(1-t)] d t+2 H(a, b) \int_{0}^{1} \frac{\varphi((b-a) t)}{t} d t .
\end{aligned}
$$

This completes the proof.

Corollary 2 If we choose $\varphi(t)=t$ in Theorem 6 , then we have the following inequalities:

$$
\begin{aligned}
& \frac{1}{2 h\left(\frac{1}{2}\right)} F\left(\frac{a+b}{2}\right)-\frac{1}{2 h\left(\frac{1}{2}\right)(b-a)} \int_{a}^{b} H(x, a+b-x) d x \\
& \supseteq \supseteq \frac{1}{b-a}(I R) \int_{a}^{b} F(x) d x \supseteq \frac{F(a)+F(b)}{2} \int_{0}^{1}[h(t)+h(1-t)] d t+H(a, b) .
\end{aligned}
$$

Corollary 3 If we choose $\varphi(t)=\frac{t^{\alpha}}{\Gamma(\alpha)}$ in Theorem 6, then we have the following inequalities for the Riemann-Liouville fractional integrals:

$$
\begin{aligned}
& \frac{1}{2 h\left(\frac{1}{2}\right)} F\left(\frac{a+b}{2}\right)-\frac{\alpha}{2 h\left(\frac{1}{2}\right)(b-a)^{\alpha}} \int_{a}^{b}(b-x)^{\alpha-1} H(x, a+b-x) d x \\
& \supseteq \frac{\Gamma(\alpha+1)}{2(b-a)^{\alpha}}\left[J_{a+}^{\alpha} F(b)+J_{b-}^{\alpha} F(a)\right] \supseteq \alpha \frac{F(a)+F(b)}{2} \int_{0}^{1} t^{\alpha-1}[h(t)+h(1-t)] d t+H(a, b) .
\end{aligned}
$$

Theorem 7 If $F, G:[a, b] \rightarrow \mathbb{R}_{\mathcal{I}}^{+}$are two interval-valued approximately $h$-convex functions such that $F(t)=[\underline{F}(t), \bar{F}(t)]$ and $G(t)=[\underline{G}(t), \bar{G}(t)]$, then we have the following inequality for the generalized fractional integrals:

$$
\begin{aligned}
& \frac{1}{2}\left[a_{+} \Im_{\varphi} F(b) G(b)+{ }_{b-} \Im_{\varphi} F(a) G(a)\right] \\
& \quad \supseteq K_{1} M(a, b)+K_{2} N(a, b)+K_{3} P(a, b) H(a, b)+\Lambda(1) H^{2}(a, b),
\end{aligned}
$$

where $M(a, b)$ and $N(a, b)$ are defined in Theorem 3 and

$$
\begin{aligned}
& P(a, b)=F(a)+G(a)+F(b)+G(b), \\
& K_{1}=\frac{1}{2} \int_{0}^{1} \frac{\varphi((b-a) t)}{t}\left[h^{2}(t)+h^{2}(1-t)\right] d t, \\
& K_{2}=\int_{0}^{1} \frac{\varphi((b-a) t)}{t} h(t) h(1-t) d t, \\
& K_{3}=\frac{1}{2} \int_{0}^{1} \frac{\varphi((b-a) t)}{t}[h(t)+h(1-t)] d t .
\end{aligned}
$$


Proof Since $F$ and $G$ are interval-valued approximately $h$-convex functions for $t \in[0,1]$, we have

$$
F(t a+(1-t) b) \supseteq h(t) F(a)+h(1-t) F(b)+H(a, b)
$$

and

$$
G(t a+(1-t) b) \supseteq h(t) G(a)+h(1-t) G(b)+H(a, b) .
$$

Multiplying (5.12) and (5.13), we get

$$
\begin{aligned}
F(t a+ & (1-t) b) G(t a+(1-t) b) \\
\supseteq & h^{2}(t) F(a) G(a)+h^{2}(1-t) F(b) G(b)+h(t) h(1-t)[F(a) G(b)+F(b) G(a)] \\
& +h(t) H(a, b)[F(a)+G(a)]+h(1-t) H(a, b)[F(b)+G(b)]+H^{2}(a, b) .
\end{aligned}
$$

Similarly, we obtain

$$
\begin{aligned}
F(t b+ & (1-t) a) G(t b+(1-t) a) \\
\supseteq & h^{2}(1-t) F(a) G(a)+h^{2}(t) F(b) G(b)+h(t) h(1-t)[F(a) G(b)+F(b) G(a)] \\
& +h(t) H(a, b)[F(b)+G(b)]+h(1-t) H(a, b)[F(a)+G(a)]+H^{2}(a, b) .
\end{aligned}
$$

Adding (5.14) and (5.15), we have the following relation:

$$
\begin{aligned}
F(t a+ & (1-t) b) G(t a+(1-t) b)+F(t b+(1-t) a) G(t b+(1-t) a) \\
\supseteq & {\left[h^{2}(t)+h^{2}(1-t)\right] M(a, b)+2 h(t) h(1-t) N(a, b) } \\
& +[h(t)+h(1-t)] P(a, b) H(a, b)+2 H^{2}(a, b) .
\end{aligned}
$$

Multiplying (5.16) by $\frac{\varphi((b-a) t)}{t}$ on both sides and integrating the resultant one with respect to $t$ over $[0,1]$, we get

$$
\begin{aligned}
(I R) & \int_{0}^{1} \frac{\varphi((b-a) t)}{t} F(t a+(1-t) b) G(t a+(1-t) b) d t \\
& +(I R) \int_{0}^{1} \frac{\varphi((b-a) t)}{t} F(t b+(1-t) a) G(t b+(1-t) a) d t \\
\supseteq & M(a, b) \int_{0}^{1} \frac{\varphi((b-a) t)}{t}\left[h^{2}(t)+h^{2}(1-t)\right] d t \\
& +2 N(a, b) \int_{0}^{1} \frac{\varphi((b-a) t)}{t} h(t) h(1-t) d t \\
& +P(a, b) H(a, b) \int_{0}^{1} \frac{\varphi((b-a) t)}{t}[h(t)+h(1-t)] d t+2 H^{2}(a, b) \int_{0}^{1} \frac{\varphi((b-a) t)}{t} d t .
\end{aligned}
$$

Using Theorem 1 in Eq. (5.17), we have

$$
\text { (IR) } \int_{0}^{1} \frac{\varphi((b-a) t)}{t} F(t a+(1-t) b) G(t a+(1-t) b) d t={ }_{a+} \Im_{\varphi} F(b) G(b)
$$


and

$$
\text { (IR) } \int_{0}^{1} \frac{\varphi((b-a) t)}{t} F(t b+(1-t) a) G(t b+(1-t) a) d t={ }_{b-} \mathfrak{I}_{\varphi} F(a) G(a) .
$$

Substituting (5.18) and (5.19) in Eq. (5.17), we have our desired result (5.11). This completes the proof.

Corollary 4 Under the assumptions of Theorem 7 with $\varphi(t)=t$, then we have the following inequality:

$$
\begin{gathered}
\frac{1}{b-a}(I R) \int_{a}^{b} F(x) G(x) d x \supseteq M(a, b) \int_{0}^{1} h^{2}(t) d t+N(a, b) \int_{0}^{1} h(t) h(1-t) d t \\
+P(a, b) H(a, b) \int_{0}^{1} h(t) d t+H^{2}(a, b) .
\end{gathered}
$$

Corollary 5 If we use $\varphi(t)=\frac{t^{\alpha}}{\Gamma(\alpha)}$ in Theorem 7 , then we have the following inequality for the Riemann-Liouville fractional integrals:

$$
\begin{aligned}
& \frac{\Gamma(\alpha+1)}{2(b-a)^{\alpha}}\left[J_{a+}^{\alpha} F(b) G(b)+J_{b-}^{\alpha} F(a) G(a)\right] \\
& \supseteq \frac{\alpha M(a, b)}{2} \int_{0}^{1} t^{\alpha-1}\left[h^{2}(t)+h^{2}(1-t)\right] d t+\alpha N(a, b) \int_{0}^{1} t^{\alpha-1} h(t) h(1-t) d t \\
& \quad+\frac{\alpha P(a, b) H(a, b)}{2} \int_{0}^{1} t^{\alpha-1}[h(t)+h(1-t)] d t+H^{2}(a, b) .
\end{aligned}
$$

Theorem 8 If $F, G:[a, b] \rightarrow \mathbb{R}_{\mathcal{I}}^{+}$are two interval-valued approximately h-convex functions such that $F(t)=[\underline{F}(t), \bar{F}(t)]$ and $G(t)=[\underline{G}(t), \bar{G}(t)]$, then we have the following inequality for the generalized fractional integrals:

$$
\begin{aligned}
& \frac{1}{2 h^{2}\left(\frac{1}{2}\right)} F\left(\frac{a+b}{2}\right) G\left(\frac{a+b}{2}\right) \\
& \quad-\frac{1}{2 \Lambda(1) h\left(\frac{1}{2}\right)}\left[(I R) \int_{a}^{b} \frac{\varphi(b-x)}{b-x}(F(x)+G(x)) H(x, a+b-x) d x\right. \\
& \left.\quad+(I R) \int_{a}^{b} \frac{\varphi(x-a)}{x-a}(F(x)+G(x)) H(a+b-x, x) d x\right] \\
& \supseteq \frac{1}{2 \Lambda(1)}\left[a+\Im_{\varphi} F(b) G(b)+{ }_{b-} \Im_{\varphi} F(a) G(a)\right] \\
& \quad+\frac{1}{2 \Lambda(1) h^{2}\left(\frac{1}{2}\right)} \int_{a}^{b} \frac{\varphi(b-x)}{b-x} H^{2}(x, a+b-x) d x \\
& \quad+\frac{1}{\Lambda(1)}\left[K_{2} M(a, b)+K_{1} N(a, b)+K_{3} P(a, b) H(a, b)\right]+H^{2}(a, b),
\end{aligned}
$$


where $M(a, b), N(a, b)$ and $K_{1}, K_{2}, K_{3}, P(a, b)$ are defined from Theorem 3 and Theorem 7 , respectively.

Proof For $t \in[0,1]$, we can write

$$
\frac{a+b}{2}=\frac{(1-t) a+t b}{2}+\frac{t a+(1-t) b}{2} .
$$

Since $F$ and $G$ are two interval-valued approximately $h$-convex functions, we have

$$
\begin{aligned}
& \frac{1}{h^{2}\left(\frac{1}{2}\right)} F\left(\frac{a+b}{2}\right) G\left(\frac{a+b}{2}\right) \\
& =\frac{1}{h^{2}\left(\frac{1}{2}\right)} F\left(\frac{(1-t) a+t b}{2}+\frac{t a+(1-t) b}{2}\right) G\left(\frac{(1-t) a+t b}{2}+\frac{t a+(1-t) b}{2}\right) \\
& \supseteq\left[F((1-t) a+t b)+F(t a+(1-t) b)+\frac{1}{h\left(\frac{1}{2}\right)} H((1-t) a+t b, t a+(1-t) b)\right] \\
& \times\left[G((1-t) a+t b)+G(t a+(1-t) b)+\frac{1}{h\left(\frac{1}{2}\right)} H((1-t) a+t b, t a+(1-t) b)\right] \\
& =[F((1-t) a+t b) G((1-t) a+t b)+F(t a+(1-t) b) G(t a+(1-t) b)] \\
& +[F((1-t) a+t b) G(t a+(1-t) b)+F(t a+(1-t) b) G((1-t) a+t b)] \\
& +\frac{1}{h\left(\frac{1}{2}\right)}[F(t a+(1-t) b) H(t a+(1-t) b, t b+(1-t) a) \\
& +F(t b+(1-t) a) H(t a+(1-t) b, t b+(1-t) a) \\
& +G(t a+(1-t) b) H(t a+(1-t) b, t b+(1-t) a) \\
& +G(t b+(1-t) a) H(t a+(1-t) b, t b+(1-t) a)] \\
& +\frac{1}{h^{2}\left(\frac{1}{2}\right)}\left[H^{2}(t a+(1-t) b, t b+(1-t) a)\right] \\
& \supseteq[F((1-t) a+t b) G((1-t) a+t b)+F(t a+(1-t) b) G(t a+(1-t) b)] \\
& +\left[h^{2}(t)+h^{2}(1-t)\right] N(a, b)+2 h(t) h(1-t) M(a, b) \\
& +[h(t)+h(1-t)] P(a, b) H(a, b)+2 H^{2}(a, b) \\
& +\frac{1}{h\left(\frac{1}{2}\right)} F(t a+(1-t) b) H(t a+(1-t) b, t b+(1-t) a) \\
& +\frac{1}{h\left(\frac{1}{2}\right)} F(t b+(1-t) a) H(t a+(1-t) b, t b+(1-t) a) \\
& +\frac{1}{h\left(\frac{1}{2}\right)} G(t a+(1-t) b) H(t a+(1-t) b, t b+(1-t) a) \\
& +\frac{1}{h\left(\frac{1}{2}\right)} G(t b+(1-t) a) H(t a+(1-t) b, t b+(1-t) a) \\
& +\frac{1}{h^{2}\left(\frac{1}{2}\right)} H^{2}(t a+(1-t) b, t b+(1-t) a) .
\end{aligned}
$$


Multiplying by $\frac{\varphi((b-a) t)}{t}$ both sides of inequality (5.23) and integrating the resultant one with respect to $t$ over $[0,1]$, we obtain

$$
\begin{aligned}
& \frac{1}{h^{2}\left(\frac{1}{2}\right)}(I R) \int_{0}^{1} \frac{\varphi((b-a) t)}{t} F\left(\frac{a+b}{2}\right) G\left(\frac{a+b}{2}\right) d t \\
& \supseteq(I R) \int_{0}^{1} \frac{\varphi((b-a) t)}{t} F((1-t) a+t b) G((1-t) a+t b) d t \\
& \quad+(I R) \int_{0}^{1} \frac{\varphi((b-a) t)}{t} F(t a+(1-t) b) G(t a+(1-t) b) d t \\
& \quad+\frac{1}{h^{2}\left(\frac{1}{2}\right)} \int_{0}^{1} \frac{\varphi((b-a) t)}{t} H^{2}(t a+(1-t) b, t b+(1-t) a) d t \\
& \quad+\frac{1}{h\left(\frac{1}{2}\right)}(I R) \int_{0}^{1} \frac{\varphi((b-a) t)}{t} F(t a+(1-t) b) H(t a+(1-t) b, t b+(1-t) a) d t \\
& \quad+\frac{1}{h\left(\frac{1}{2}\right)}(I R) \int_{0}^{1} \frac{\varphi((b-a) t)}{t} F(t b+(1-t) a) H(t a+(1-t) b, t b+(1-t) a) d t \\
& \quad+\frac{1}{h\left(\frac{1}{2}\right)}(I R) \int_{0}^{1} \frac{\varphi((b-a) t)}{t} G(t a+(1-t) b) H(t a+(1-t) b, t b+(1-t) a) d t \\
& \quad+\frac{1}{h\left(\frac{1}{2}\right)}(I R) \int_{0}^{1} \frac{\varphi((b-a) t)}{t} G(t b+(1-t) a) H(t a+(1-t) b, t b+(1-t) a) d t \\
& \quad+N(a, b) \int_{0}^{1} \frac{\varphi((b-a) t)}{t}\left[h^{2}(t)+h^{2}(1-t)\right] d t \\
& \quad+2 M(a, b) \int_{0}^{1} \frac{\varphi((b-a) t)}{t} h(t) h(1-t) d t \\
& +P(a, b) H(a, b) \int_{0}^{1} \frac{\varphi((b-a) t)}{t}[h(t)+h(1-t)] d t+2 H^{2}(a, b) \int_{0}^{1} \frac{\varphi((b-a) t)}{t} d t
\end{aligned}
$$

By changing the variable of integration we achieved the desired inequality (5.22).

Corollary 6 Under the assumptions of Theorem 8 with $\varphi(t)=t$, then we have the following inequality:

$$
\begin{aligned}
& \frac{1}{2 h^{2}\left(\frac{1}{2}\right)} F\left(\frac{a+b}{2}\right) G\left(\frac{a+b}{2}\right) \\
& \quad-\frac{1}{(b-a) h\left(\frac{1}{2}\right)}(I R) \int_{a}^{b}[F(x)+G(x)] H(x, a+b-x) d x \\
& \supseteq \frac{1}{b-a}(I R) \int_{a}^{b} F(x) G(x) d x+\frac{1}{2(b-a) h^{2}\left(\frac{1}{2}\right)} \int_{a}^{b} H^{2}(x, a+b-x) d x \\
& \quad+\left[M(a, b) \int_{0}^{1} h(t) h(1-t) d t+N(a, b) \int_{0}^{1} h^{2}(t) d t\right. \\
& \left.\quad+P(a, b) H(a, b) \int_{0}^{1} h(t) d t\right]+H^{2}(a, b) .
\end{aligned}
$$


Corollary 7 Under the assumptions of Theorem 8 with $\varphi(t)=\frac{t^{\alpha}}{\Gamma(\alpha)}$, then we have the following inequality for the Riemann-Liouville fractional integrals:

$$
\begin{aligned}
& \frac{1}{2 h^{2}\left(\frac{1}{2}\right)} F\left(\frac{a+b}{2}\right) G\left(\frac{a+b}{2}\right) \\
& \quad-\frac{\alpha}{2(b-a)^{\alpha} h\left(\frac{1}{2}\right)}\left[(I R) \int_{a}^{b}(b-x)^{\alpha-1}[F(x)+G(x)] H(x, a+b-x) d x\right. \\
& \left.\quad+(I R) \int_{a}^{b}(x-a)^{\alpha-1}[F(x)+G(x)] H(a+b-x, x) d x\right] \\
& \supseteq \frac{\Gamma(\alpha+1)}{2(b-a)^{\alpha}}\left[J_{a+}^{\alpha} F(b) G(b)+F(a) G(a)\right] \\
& \quad+\frac{\alpha}{2(b-a)^{\alpha} h^{2}\left(\frac{1}{2}\right)} \int_{a}^{b}(b-x)^{\alpha-1} H^{2}(x, a+b-x) d x \\
& \quad+\alpha\left[M(a, b) \int_{0}^{1} t^{\alpha-1} h(t) h(1-t) d t+\frac{N(a, b)}{2} \int_{0}^{1} t^{\alpha-1}\left[h^{2}(t)+h^{2}(1-t)\right] d t\right. \\
& \left.\quad+\frac{P(a, b) H(a, b)}{2} \int_{0}^{1} t^{\alpha-1}[h(t)+h(1-t)] d t\right]+H^{2}(a, b) .
\end{aligned}
$$

Theorem 9 If $F:[a, b] \rightarrow \mathbb{R}_{\mathcal{I}}^{+}$is an interval-valued approximately $h$-convex function such that $F(t)=[\underline{F}(t), \bar{F}(t)]$, then we have the following inequalities for the generalized fractional integrals:

$$
\begin{aligned}
& \frac{1}{2 h\left(\frac{1}{2}\right)} F\left(\frac{a+b}{2}\right)-\frac{1}{2 \psi(1) h\left(\frac{1}{2}\right)} \int_{\frac{a+b}{2}}^{b} \frac{\varphi(b-x)}{b-x} H(x, a+b-x) d x \\
& \supseteq \frac{1}{2 \psi(1)}\left[\left(\frac{a+b}{2}\right)^{+} \Im_{\varphi} F(b)+\left(\frac{a+b}{2}\right)^{-} \Im_{\varphi} F(a)\right] \\
& \supseteq \frac{F(a)+F(b)}{2 \psi(1)} \int_{0}^{1} \frac{\varphi\left(\frac{(b-a)}{2} t\right)}{t}\left[h\left(\frac{2-t}{2}\right)+h\left(\frac{t}{2}\right)\right] d t+H(a, b) .
\end{aligned}
$$

Proof Since $F$ is interval-valued approximately $h$-convex function on $[a, b]$, we have

$$
\frac{1}{h\left(\frac{1}{2}\right)} F\left(\frac{x+y}{2}\right) \supseteq F(x)+F(y)+\frac{1}{h\left(\frac{1}{2}\right)} H(x, y) .
$$

For $x=\frac{t}{2} a+\frac{2-t}{2} b$ and $y=\frac{2-t}{2} a+\frac{t}{2} b$, we get

$$
\begin{aligned}
& \frac{1}{h\left(\frac{1}{2}\right)} F\left(\frac{a+b}{2}\right) \\
& \quad \supseteq F\left(\frac{t}{2} a+\frac{2-t}{2} b\right)+F\left(\frac{2-t}{2} a+\frac{t}{2} b\right)+\frac{1}{h\left(\frac{1}{2}\right)} H\left(\frac{t}{2} a+\frac{2-t}{2} b, \frac{2-t}{2} a+\frac{t}{2} b\right) .
\end{aligned}
$$

Multiplying by $\frac{\varphi\left(\frac{(b-a)}{2} t\right)}{t}$ both sides of inequality (5.27) and integrating the resultant one with respect to $t$ over $[0,1]$, we obtain

$$
\frac{1}{h\left(\frac{1}{2}\right)} F\left(\frac{a+b}{2}\right) \int_{0}^{1} \frac{\varphi\left(\frac{(b-a)}{2} t\right)}{t} d t
$$




$$
\begin{aligned}
& \supseteq(I R) \int_{0}^{1} \frac{\varphi\left(\frac{(b-a)}{2} t\right)}{t} F\left(\frac{t}{2} a+\frac{2-t}{2} b\right) d t+(I R) \int_{0}^{1} \frac{\varphi\left(\frac{(b-a)}{2} t\right)}{t} F\left(\frac{2-t}{2} a+\frac{t}{2} b\right) d t \\
& \quad+\frac{1}{h\left(\frac{1}{2}\right)} \int_{0}^{1} \frac{\varphi\left(\frac{(b-a)}{2} t\right)}{t} H\left(\frac{t}{2} a+\frac{2-t}{2} b, \frac{2-t}{2} a+\frac{t}{2} b\right) d t .
\end{aligned}
$$

Using Theorem 1 and Eq. (5.28), we have

$$
\text { (IR) } \begin{aligned}
& \int_{0}^{1} \frac{\varphi\left(\frac{(b-a)}{2} t\right)}{t} F\left(\frac{t}{2} a+\frac{2-t}{2} b\right) d t \\
= & {\left[(R) \int_{0}^{1} \frac{\varphi\left(\frac{(b-a)}{2} t\right)}{t} \underline{F}\left(\frac{t}{2} a+\frac{2-t}{2} b\right) d t,(R) \int_{0}^{1} \frac{\varphi\left(\frac{(b-a)}{2} t\right)}{t} \bar{F}\left(\frac{t}{2} a+\frac{2-t}{2} b\right) d t\right] } \\
= & {\left[(R) \int_{\frac{a+b}{2}}^{b} \frac{\varphi(b-u)}{b-u} \underline{F}(u) d u,(R) \int_{\frac{a+b}{2}}^{b} \frac{\varphi(b-u)}{b-u} \bar{F}(u) d u\right] } \\
= & {\left[\left(\frac{a+b}{2}\right)^{+} I_{\varphi} \underline{F}(b)_{\left.{ }_{\left(\frac{a+b}{2}\right)^{+}} I_{\varphi} \bar{F}(b)\right]}\right.} \\
= & { }_{\left(\frac{a+b}{2}\right)^{+}} \Im_{\varphi} F(b) .
\end{aligned}
$$

Similarly, we get

$$
\text { (IR) } \int_{0}^{1} \frac{\varphi\left(\frac{(b-a)}{2} t\right)}{t} F\left(\frac{2-t}{2} a+\frac{t}{2} b\right) d t=\left[{ }_{\left(\frac{a+b}{2}\right)^{-}} I_{\varphi} \underline{F}(a),_{\left(\frac{a+b}{2}\right)^{-}} I_{\varphi} \bar{F}(a)\right]={ }_{\left(\frac{a+b}{2}\right)^{-}} \mathfrak{I}_{\varphi} F(a) .
$$

Hence, we proved the first inequality. To prove the second inequality of (5.26), first we note that, since $F$ is an interval-valued approximately $h$-convex function, we have

$$
F\left(\frac{2-t}{2} a+\frac{t}{2} b\right) \supseteq h\left(\frac{2-t}{2}\right) F(a)+h\left(\frac{t}{2}\right) F(b)+H(a, b)
$$

and

$$
F\left(\frac{t}{2} a+\frac{2-t}{2} b\right) \supseteq h\left(\frac{t}{2}\right) F(a)+h\left(\frac{2-t}{2}\right) F(b)+H(a, b) .
$$

Adding (5.29) and (5.30), we get

$$
\begin{aligned}
& F\left(\frac{2-t}{2} a+\frac{t}{2} b\right)+F\left(\frac{t}{2} a+\frac{2-t}{2} b\right) \\
& \quad \supseteq[F(a)+F(b)]\left[h\left(\frac{2-t}{2}\right)+h\left(\frac{t}{2}\right)\right]+2 H(a, b) .
\end{aligned}
$$

Multiplying by $\frac{\varphi\left(\frac{(b-a)}{2} t\right)}{t}$ both sides of inequality (5.31) and integrating the resultant one with respect to $t$ over $[0,1]$, we obtain

$$
\text { (IR) } \int_{0}^{1} \frac{\varphi\left(\frac{(b-a)}{2} t\right)}{t} F\left(\frac{2-t}{2} a+\frac{t}{2} b\right) d t+(I R) \int_{0}^{1} \frac{\varphi\left(\frac{(b-a)}{2} t\right)}{t} F\left(\frac{t}{2} a+\frac{2-t}{2} b\right) d t
$$




$$
\begin{aligned}
\supseteq & {[F(a)+F(b)](I R) \int_{0}^{1} \frac{\varphi\left(\frac{(b-a)}{2} t\right)}{t}\left[h\left(\frac{2-t}{2}\right)+h\left(\frac{t}{2}\right)\right] d t } \\
& +2 H(a, b) \int_{0}^{1} \frac{\varphi\left(\frac{(b-a)}{2} t\right)}{t} d t .
\end{aligned}
$$

By changing the variables of integration we have the second inequality of (5.26).

Corollary 8 If we choose $\varphi(t)=t$ in Theorem 9, then we have the following inequalities:

$$
\begin{aligned}
& \frac{1}{2 h\left(\frac{1}{2}\right)} F\left(\frac{a+b}{2}\right)-\frac{1}{(b-a) h\left(\frac{1}{2}\right)} \int_{\frac{a+b}{2}}^{b} H(x, a+b-x) d x \\
& \supseteq \supseteq \frac{1}{b-a}(I R) \int_{a}^{b} F(x) d x \supseteq \frac{F(a)+F(b)}{2} \int_{0}^{1}\left[h\left(\frac{2-t}{2}\right)+h\left(\frac{t}{2}\right)\right] d t+H(a, b) .
\end{aligned}
$$

Corollary 9 Taking $\varphi(t)=\frac{t^{\alpha}}{\Gamma(\alpha)}$ in Theorem 9, then we have the following inequalities for the interval-valued fractional operators:

$$
\begin{aligned}
& \frac{1}{2 h\left(\frac{1}{2}\right)} F\left(\frac{a+b}{2}\right)-\frac{\alpha 2^{\alpha-1}}{(b-a)^{\alpha} h\left(\frac{1}{2}\right)} \int_{\frac{a+b}{2}}^{b}(b-x)^{\alpha-1} H(x, a+b-x) d x \\
& \supseteq \frac{2^{\alpha-1} \Gamma(\alpha+1)}{(b-a)^{\alpha}}\left[J_{\left(\frac{a+b}{2}\right)+}^{\alpha} F(b)+J_{\left(\frac{a+b}{2}\right)-}^{\alpha} F(a)\right] \\
& \supseteq \frac{\alpha[F(a)+F(b)]}{2} \int_{0}^{1} t^{\alpha-1}\left[h\left(\frac{2-t}{2}\right)+h\left(\frac{t}{2}\right)\right] d t+H(a, b) .
\end{aligned}
$$

Theorem 10 If $F, G:[a, b] \rightarrow \mathbb{R}_{\mathcal{I}}^{+}$are two interval-valued approximately $h$-convex functions such that $F(t)=[\underline{F}(t), \bar{F}(t)]$ and $G(t)=[\underline{G}(t), \bar{G}(t)]$, then we have the following inequality for the generalized fractional integrals:

$$
\begin{aligned}
& {\left[\frac{\left(\frac{a+b}{2}\right)^{+}}{\Im_{\varphi}} F(b) G(b)+_{\left(\frac{a+b}{2}\right)^{-}} \Im_{\varphi} F(a) G(a)\right]} \\
& \supseteq \supseteq K_{4} M(a, b)+K_{5} N(a, b)+H(a, b) P(a, b) K_{6}+2 \psi(1) H^{2}(a, b),
\end{aligned}
$$

where $M(a, b)$ and $N(a, b)$ are defined in Theorem 3 and

$$
\begin{aligned}
& K_{4}=\int_{0}^{1} \frac{\varphi\left(\frac{(b-a)}{2} t\right)}{t}\left[h^{2}\left(\frac{t}{2}\right)+h^{2}\left(\frac{2-t}{2}\right)\right] d t, \\
& K_{5}=2 \int_{0}^{1} \frac{\varphi\left(\frac{(b-a)}{2} t\right)}{t} h\left(\frac{t}{2}\right) h\left(\frac{2-t}{2}\right) d t, \\
& K_{6}=\int_{0}^{1} \frac{\varphi\left(\frac{(b-a)}{2} t\right)}{t}\left[h\left(\frac{t}{2}\right)+h\left(\frac{2-t}{2}\right)\right] d t .
\end{aligned}
$$

Proof Since $F$ and $G$ are two interval-valued approximately $h$-convex functions,

$$
F\left(\frac{2-t}{2} a+\frac{t}{2} b\right) \supseteq h\left(\frac{2-t}{2}\right) F(a)+h\left(\frac{t}{2}\right) F(b)+H(a, b)
$$


and

$$
G\left(\frac{2-t}{2} a+\frac{t}{2} b\right) \supseteq h\left(\frac{2-t}{2}\right) G(a)+h\left(\frac{t}{2}\right) G(b)+H(a, b) .
$$

Multiplying (5.35) and (5.36), we have

$$
\begin{aligned}
F( & \left.\frac{2-t}{2} a+\frac{t}{2} b\right) G\left(\frac{2-t}{2} a+\frac{t}{2} b\right) \\
\supseteq & h^{2}\left(\frac{2-t}{2}\right) F(a) G(a)+h^{2}\left(\frac{t}{2}\right) F(b) G(b)+h\left(\frac{2-t}{2}\right) h\left(\frac{t}{2}\right)[F(a) G(b)+F(b) G(a)] \\
& +h\left(\frac{t}{2}\right) H(a, b)[F(b)+G(b)]+h\left(\frac{2-t}{2}\right) H(a, b)[F(a)+G(a)]+H^{2}(a, b) .
\end{aligned}
$$

Similarly, we get

$$
\begin{aligned}
F\left(\frac{t}{2} a+\frac{2-t}{2} b\right) G\left(\frac{t}{2} a+\frac{2-t}{2} b\right) \\
\supseteq h^{2}\left(\frac{t}{2}\right) F(a) G(a)+h^{2}\left(\frac{2-t}{2}\right) F(b) G(b)+h\left(\frac{t}{2}\right) h\left(\frac{2-t}{2}\right)[F(a) G(b)+F(b) G(a)] \\
\quad+h\left(\frac{2-t}{2}\right) H(a, b)[F(b)+G(b)]+h\left(\frac{t}{2}\right) H(a, b)[F(a)+G(a)]+H^{2}(a, b) .
\end{aligned}
$$

Adding (5.37) and (5.38), we obtain the following relation:

$$
\begin{aligned}
F( & \left.\frac{2-t}{2} a+\frac{t}{2} b\right) G\left(\frac{2-t}{2} a+\frac{t}{2} b\right)+F\left(\frac{t}{2} a+\frac{2-t}{2} b\right) G\left(\frac{t}{2} a+\frac{2-t}{2} b\right) \\
\supseteq & h^{2}\left(\frac{2-t}{2}\right)[F(a) G(a)+F(b) G(b)] \\
& +h^{2}\left(\frac{t}{2}\right)[F(a) G(a)+F(b) G(b)]+2 h\left(\frac{t}{2}\right) h\left(\frac{2-t}{2}\right)[F(a) G(b)+F(b) G(a)] \\
& +H(a, b)\left[h\left(\frac{t}{2}\right)+h\left(\frac{2-t}{2}\right)\right][F(a)+F(b)+G(a)+G(b)]+2 H^{2}(a, b) \\
= & {\left[h^{2}\left(\frac{2-t}{2}\right)+h^{2}\left(\frac{t}{2}\right)\right] M(a, b)+2 h\left(\frac{t}{2}\right) h\left(\frac{2-t}{2}\right) N(a, b) } \\
& +H(a, b) P(a, b)\left[h\left(\frac{t}{2}\right)+h\left(\frac{2-t}{2}\right)\right]+2 H^{2}(a, b) .
\end{aligned}
$$

Multiplying by $\frac{\varphi\left(\frac{(b-a)}{2} t\right)}{t}$ both sides of inequality (5.39) and integrating the resultant one with respect to $t$ over $[0,1]$, we have

$$
\begin{aligned}
& \text { (IR) } \int_{0}^{1} \frac{\varphi\left(\frac{(b-a)}{2} t\right)}{t} F\left(\frac{2-t}{2} a+\frac{t}{2} b\right) G\left(\frac{2-t}{2} a+\frac{t}{2} b\right) d t \\
& \quad+(I R) \int_{0}^{1} \frac{\varphi\left(\frac{(b-a)}{2} t\right)}{t} F\left(\frac{t}{2} a+\frac{2-t}{2} b\right) G\left(\frac{t}{2} a+\frac{2-t}{2} b\right) d t \\
& \supseteq M(a, b) \int_{0}^{1} \frac{\varphi\left(\frac{(b-a)}{2} t\right)}{t}\left[h^{2}\left(\frac{2-t}{2}\right)+h^{2}\left(\frac{t}{2}\right)\right] d t
\end{aligned}
$$




$$
\begin{aligned}
& +2 N(a, b) \int_{0}^{1} \frac{\varphi\left(\frac{(b-a)}{2} t\right)}{t} h\left(\frac{t}{2}\right) h\left(\frac{2-t}{2}\right) d t \\
& +H(a, b) P(a, b) \int_{0}^{1} \frac{\varphi\left(\frac{(b-a)}{2} t\right)}{t}\left[h\left(\frac{t}{2}\right)+h\left(\frac{2-t}{2}\right)\right] d t \\
& +2 H^{2}(a, b) \int_{0}^{1} \frac{\varphi\left(\frac{(b-a)}{2} t\right)}{t} d t
\end{aligned}
$$

By using Theorem 1 in Eq. (5.40), we obtain our required inequality.

Corollary 10 Taking $\varphi(t)=t$ in Theorem 10, then we have the following inequality:

$$
\begin{aligned}
& \frac{2}{b-a}(I R) \int_{a}^{b} F(x) G(x) d x \\
& \supseteq M(a, b) \int_{0}^{1}\left[h^{2}\left(\frac{t}{2}\right)+h^{2}\left(\frac{2-t}{2}\right)\right] d t+2 N(a, b) \int_{0}^{1} h\left(\frac{t}{2}\right) h\left(\frac{2-t}{2}\right) d t \\
& \quad+H(a, b) P(a, b) \int_{0}^{1}\left[h\left(\frac{t}{2}\right)+h\left(\frac{2-t}{2}\right)\right] d t+2 H^{2}(a, b) .
\end{aligned}
$$

Corollary 11 Taking $\varphi(t)=\frac{t^{\alpha}}{\Gamma(\alpha)}$ in Theorem 10, then we have the following inequality for the interval-valued fractional operators:

$$
\begin{aligned}
& \frac{2^{\alpha} \Gamma(\alpha+1)}{(b-a)^{\alpha}}\left[J_{\left(\frac{a+b}{2}\right)+}^{\alpha} F(b) G(b)+J_{\left(\frac{a+b}{2}\right)-}^{\alpha} F(a) G(a)\right] \\
& \supseteq \alpha M(a, b) \int_{0}^{1} t^{\alpha-1}\left[h^{2}\left(\frac{t}{2}\right)+h^{2}\left(\frac{2-t}{2}\right)\right] d t \\
& \quad+2 \alpha N(a, b) \int_{0}^{1} t^{\alpha-1} h\left(\frac{t}{2}\right) h\left(\frac{2-t}{2}\right) d t \\
& \quad+\alpha H(a, b) P(a, b) \int_{0}^{1} t^{\alpha-1}\left[h\left(\frac{t}{2}\right)+h\left(\frac{2-t}{2}\right)\right] d t+2 H^{2}(a, b) .
\end{aligned}
$$

Theorem 11 If $F, G:[a, b] \rightarrow \mathbb{R}_{\mathcal{I}}^{+}$are two interval-valued approximately h-convex functions such that $F(t)=[\underline{F}(t), \bar{F}(t)]$ and $G(t)=[\underline{G}(t), \bar{G}(t)]$, then we have the following inequality for the generalized fractional integrals:

$$
\begin{aligned}
& \frac{1}{h^{2}\left(\frac{1}{2}\right)} F\left(\frac{a+b}{2}\right) G\left(\frac{a+b}{2}\right) \\
& \quad-\frac{1}{h\left(\frac{1}{2}\right) \psi(1)}\left[(I R) \int_{\frac{a+b}{2}}^{b} \frac{\varphi(b-x)}{b-x}[F(x)+G(x)] H(x, a+b-x) d x\right. \\
& \left.\quad+(I R) \int_{a}^{\frac{a+b}{2}} \frac{\varphi(x-a)}{x-a}[F(x)+G(x)] H(a+b-x, x) d x\right] \\
& \supseteq \frac{1}{\psi(1)}\left[\left(\frac{a+b}{2}\right)^{+} \Im_{\varphi} F(b) G(b)+\left(\frac{a+b}{2}\right)^{-} \Im_{\varphi} F(a) G(a)\right] \\
& \quad+\frac{1}{\psi(1) h^{2}\left(\frac{1}{2}\right)} \int_{a}^{\frac{a+b}{2}} \frac{\varphi(x-a)}{x-a} H^{2}(x, a+b-x) d x \\
& \quad+\frac{1}{\psi(1)}\left[K_{5} M(a, b)+K_{4} N(a, b)+K_{6} P(a, b) H(a, b)\right]+2 H^{2}(a, b)
\end{aligned}
$$


where $M(a, b), N(a, b)$ and $K_{4}, K_{5}, K_{6}$ are defined in Theorem 3 and Theorem 10, respectively.

Proof Since $F$ and $G$ are two interval-valued approximately $h$-convex functions on $[a, b]$, we have

$$
\frac{1}{h\left(\frac{1}{2}\right)} F\left(\frac{x+y}{2}\right) \supseteq F(x)+F(y)+\frac{1}{h\left(\frac{1}{2}\right)} H(x, y) .
$$

For $x=\frac{2-t}{2} a+\frac{t}{2} b$ and $y=\frac{t}{2} a+\frac{2-t}{2} b$, we obtain

$$
\begin{aligned}
\frac{1}{h\left(\frac{1}{2}\right)} F\left(\frac{a+b}{2}\right) \supseteq & F\left(\frac{2-t}{2} a+\frac{t}{2} b\right)+F\left(\frac{t}{2} a+\frac{2-t}{2} b\right) \\
& +\frac{1}{h\left(\frac{1}{2}\right)} H\left(\frac{2-t}{2} a+\frac{t}{2} b, \frac{t}{2} a+\frac{2-t}{2} b\right) .
\end{aligned}
$$

Similarly, we get

$$
\begin{aligned}
\frac{1}{h\left(\frac{1}{2}\right)} G\left(\frac{a+b}{2}\right) \supseteq & G\left(\frac{2-t}{2} a+\frac{t}{2} b\right)+G\left(\frac{t}{2} a+\frac{2-t}{2} b\right) \\
& +\frac{1}{h\left(\frac{1}{2}\right)} H\left(\frac{2-t}{2} a+\frac{t}{2} b, \frac{t}{2} a+\frac{2-t}{2} b\right) .
\end{aligned}
$$

Multiplying the inequalities (5.45) and (5.46), we obtain

$$
\begin{aligned}
\frac{1}{h^{2}\left(\frac{1}{2}\right)} & F\left(\frac{a+b}{2}\right) G\left(\frac{a+b}{2}\right) \\
\supseteq & F\left(\frac{2-t}{2} a+\frac{t}{2} b\right) G\left(\frac{2-t}{2} a+\frac{t}{2} b\right) \\
& +F\left(\frac{t}{2} a+\frac{2-t}{2} b\right) G\left(\frac{t}{2} a+\frac{2-t}{2} b\right) \\
& +F\left(\frac{2-t}{2} a+\frac{t}{2} b\right) G\left(\frac{t}{2} a+\frac{2-t}{2} b\right) \\
& +F\left(\frac{t}{2} a+\frac{2-t}{2} b\right) G\left(\frac{2-t}{2} a+\frac{t}{2} b\right) \\
& +\frac{1}{h^{2}\left(\frac{1}{2}\right)} H^{2}\left(\frac{2-t}{2} a+\frac{t}{2} b, \frac{t}{2} a+\frac{2-t}{2} b\right) \\
& +\frac{1}{h\left(\frac{1}{2}\right)} F\left(\frac{2-t}{2} a+\frac{t}{2} b\right) H\left(\frac{2-t}{2} a+\frac{t}{2} b, \frac{t}{2} a+\frac{2-t}{2} b\right) \\
& +\frac{1}{h\left(\frac{1}{2}\right)} F\left(\frac{t}{2} a+\frac{2-t}{2} b\right) H\left(\frac{2-t}{2} a+\frac{t}{2} b, \frac{t}{2} a+\frac{2-t}{2} b\right) \\
& +\frac{1}{h\left(\frac{1}{2}\right)} G\left(\frac{2-t}{2} a+\frac{t}{2} b\right) H\left(\frac{2-t}{2} a+\frac{t}{2} b, \frac{t}{2} a+\frac{2-t}{2} b\right) \\
& +\frac{1}{h\left(\frac{1}{2}\right)} G\left(\frac{t}{2} a+\frac{2-t}{2} b\right) H\left(\frac{2-t}{2} a+\frac{t}{2} b, \frac{t}{2} a+\frac{2-t}{2} b\right) \\
\supseteq & F\left(\frac{2-t}{2} a+\frac{t}{2} b\right) G\left(\frac{2-t}{2} a+\frac{t}{2} b\right)+F\left(\frac{t}{2} a+\frac{2-t}{2} b\right) G\left(\frac{t}{2} a+\frac{2-t}{2} b\right)
\end{aligned}
$$




$$
\begin{aligned}
& +\left[h\left(\frac{2-t}{2}\right) F(a)+h\left(\frac{t}{2}\right) F(b)+H(a, b)\right] \\
& \times\left[h\left(\frac{t}{2}\right) G(a)+h\left(\frac{2-t}{2}\right) G(b)+H(a, b)\right] \\
& +\left[h\left(\frac{t}{2}\right) F(a)+h\left(\frac{2-t}{2}\right) F(b)+H(a, b)\right] \\
& \times\left[h\left(\frac{2-t}{2}\right) G(a)+h\left(\frac{t}{2}\right) G(b)+H(a, b)\right] \\
& +\frac{1}{h^{2}\left(\frac{1}{2}\right)} H^{2}\left(\frac{2-t}{2} a+\frac{t}{2} b, \frac{t}{2} a+\frac{2-t}{2} b\right) \\
& +\frac{1}{h\left(\frac{1}{2}\right)} F\left(\frac{2-t}{2} a+\frac{t}{2} b\right) H\left(\frac{2-t}{2} a+\frac{t}{2} b, \frac{t}{2} a+\frac{2-t}{2} b\right) \\
& +\frac{1}{h\left(\frac{1}{2}\right)} F\left(\frac{t}{2} a+\frac{2-t}{2} b\right) H\left(\frac{2-t}{2} a+\frac{t}{2} b, \frac{t}{2} a+\frac{2-t}{2} b\right) \\
& +\frac{1}{h\left(\frac{1}{2}\right)} G\left(\frac{2-t}{2} a+\frac{t}{2} b\right) H\left(\frac{2-t}{2} a+\frac{t}{2} b, \frac{t}{2} a+\frac{2-t}{2} b\right) \\
& +\frac{1}{h\left(\frac{1}{2}\right)} G\left(\frac{t}{2} a+\frac{2-t}{2} b\right) H\left(\frac{2-t}{2} a+\frac{t}{2} b, \frac{t}{2} a+\frac{2-t}{2} b\right) \\
& =F\left(\frac{2-t}{2} a+\frac{t}{2} b\right) G\left(\frac{2-t}{2} a+\frac{t}{2} b\right)+F\left(\frac{t}{2} a+\frac{2-t}{2} b\right) G\left(\frac{t}{2} a+\frac{2-t}{2} b\right) \\
& +2 M(a, b) h\left(\frac{2-t}{2}\right) h\left(\frac{t}{2}\right)+\left[h^{2}\left(\frac{2-t}{2}\right)+h^{2}\left(\frac{t}{2}\right)\right] N(a, b) \\
& +\left[h\left(\frac{t}{2}\right)+h\left(\frac{2-t}{2}\right)\right] P(a, b) H(a, b)+\frac{1}{h^{2}\left(\frac{1}{2}\right)} H^{2}\left(\frac{2-t}{2} a+\frac{t}{2} b, \frac{t}{2} a+\frac{2-t}{2} b\right) \\
& +\frac{1}{h\left(\frac{1}{2}\right)} F\left(\frac{2-t}{2} a+\frac{t}{2} b\right) H\left(\frac{2-t}{2} a+\frac{t}{2} b, \frac{t}{2} a+\frac{2-t}{2} b\right) \\
& +\frac{1}{h\left(\frac{1}{2}\right)} F\left(\frac{t}{2} a+\frac{2-t}{2} b\right) H\left(\frac{2-t}{2} a+\frac{t}{2} b, \frac{t}{2} a+\frac{2-t}{2} b\right) \\
& +\frac{1}{h\left(\frac{1}{2}\right)} G\left(\frac{2-t}{2} a+\frac{t}{2} b\right) H\left(\frac{2-t}{2} a+\frac{t}{2} b, \frac{t}{2} a+\frac{2-t}{2} b\right) \\
& +\frac{1}{h\left(\frac{1}{2}\right)} G\left(\frac{t}{2} a+\frac{2-t}{2} b\right) H\left(\frac{2-t}{2} a+\frac{t}{2} b, \frac{t}{2} a+\frac{2-t}{2} b\right)+2 H^{2}(a, b) \text {. }
\end{aligned}
$$

Multiplying by $\frac{\varphi\left(\frac{(b-a)}{2} t\right)}{t}$ both sides of inequality (5.47) and integrating the resultant one with respect to $t$ over $[0,1]$, we obtain our result (5.43).

Corollary 12 Taking $\varphi(t)=t$ in Theorem 11, then we have the following inequality:

$$
\begin{aligned}
& \frac{1}{h^{2}\left(\frac{1}{2}\right)} F\left(\frac{a+b}{2}\right) G\left(\frac{a+b}{2}\right) \\
& \quad-\frac{2}{(b-a) h\left(\frac{1}{2}\right)}\left[(I R) \int_{\frac{a+b}{2}}^{b}[F(x)+G(x)] H(x, a+b-x) d x\right. \\
& \left.\quad+(I R) \int_{a}^{\frac{a+b}{2}}[F(x)+G(x)] H(a+b-x, x) d x\right]
\end{aligned}
$$




$$
\begin{aligned}
& \supseteq \frac{2}{b-a}(I R) \int_{a}^{b} F(x) G(x) d x+\frac{2}{(b-a) h^{2}\left(\frac{1}{2}\right)} \int_{\frac{a+b}{2}}^{b} H^{2}(x, a+b-x) d x \\
& \quad+2 M(a, b) \int_{0}^{1} h\left(\frac{t}{2}\right) h\left(\frac{2-t}{2}\right) d t+N(a, b) \int_{0}^{1}\left[h^{2}\left(\frac{t}{2}\right)+h^{2}\left(\frac{2-t}{2}\right)\right] d t \\
& \quad+P(a, b) H(a, b) \int_{0}^{1}\left[h\left(\frac{t}{2}\right)+h\left(\frac{2-t}{2}\right)\right] d t+2 H^{2}(a, b) .
\end{aligned}
$$

Corollary 13 Taking $\varphi(t)=\frac{t^{\alpha}}{\Gamma(\alpha)}$ in Theorem 11, then we have the following inequality for the interval-valued fractional operators:

$$
\begin{aligned}
\frac{1}{h^{2}\left(\frac{1}{2}\right)} & F\left(\frac{a+b}{2}\right) G\left(\frac{a+b}{2}\right) \\
& -\frac{2^{\alpha} \alpha}{(b-a)^{\alpha} h\left(\frac{1}{2}\right)}\left[(I R) \int_{\frac{a+b}{2}}^{b}(b-x)^{\alpha-1}[F(x)+G(x)] H(x, a+b-x) d x\right. \\
& \left.+(I R) \int_{a}^{\frac{a+b}{2}}(x-a)^{\alpha-1}[F(x)+G(x)] H(a+b-x, x) d x\right] \\
\supseteq & \frac{2^{\alpha} \Gamma(\alpha+1)}{(b-a)^{\alpha}}\left[J_{\left(\frac{a+b}{2}\right)+}^{\alpha} F(b) G(b)+J_{\left(\frac{a+b}{2}\right)-}^{\alpha} F(a) G(a)\right] \\
& +\frac{\alpha 2^{\alpha}}{(b-a)^{\alpha} h^{2}\left(\frac{1}{2}\right)} \int_{\frac{a+b}{2}}^{b}(b-x)^{\alpha-1} H^{2}(x, a+b-x) d x \\
& +\alpha\left\{2 M(a, b) \int_{0}^{1} t^{\alpha-1} h\left(\frac{t}{2}\right) h\left(\frac{2-t}{2}\right) d t\right. \\
& +N(a, b) \int_{0}^{1} t^{\alpha-1}\left[h^{2}\left(\frac{t}{2}\right)+h^{2}\left(\frac{2-t}{2}\right)\right] d t \\
& \left.+P(a, b) H(a, b) \int_{0}^{1} t^{\alpha-1}\left[h\left(\frac{t}{2}\right)+h\left(\frac{2-t}{2}\right)\right] d t\right\}+2 H^{2}(a, b) .
\end{aligned}
$$

Theorem 12 If $F:[a, b] \rightarrow \mathbb{R}_{\mathcal{I}}^{+}$is interval-valued approximately h-convex function such that $F(t)=[\underline{F}(t), \bar{F}(t)]$, then we have the following inequalities for the generalized fractional integrals:

$$
\begin{aligned}
& \frac{1}{2 h\left(\frac{1}{2}\right)} F\left(\frac{a+b}{2}\right)-\frac{1}{2 \psi(1) h\left(\frac{1}{2}\right)} \int_{\frac{a+b}{2}}^{b} \frac{\varphi\left(x-\frac{a+b}{2}\right)}{x-\frac{a+b}{2}} H(x, a+b-x) d x \\
& \supseteq \frac{1}{2 \psi(1)}\left[{ }_{a+} \mathfrak{I}_{\varphi} F\left(\frac{a+b}{2}\right)+{ }_{b-} \mathfrak{I}_{\varphi} F\left(\frac{a+b}{2}\right)\right] \\
& \supseteq \frac{F(a)+F(b)}{2 \psi(1)} \int_{0}^{1} \frac{\varphi\left(\frac{(b-a)}{2} t\right)}{t}\left[h\left(\frac{1+t}{2}\right)+h\left(\frac{1-t}{2}\right)\right] d t+H(a, b) .
\end{aligned}
$$

Proof Since $F$ is an interval-valued approximately $h$-convex function on $[a, b]$, we have

$$
\frac{1}{h\left(\frac{1}{2}\right)} F\left(\frac{x+y}{2}\right) \supseteq F(x)+F(y)+\frac{1}{h\left(\frac{1}{2}\right)} H(x, y) .
$$


For $x=\frac{1-t}{2} a+\frac{1+t}{2} b$ and $y=\frac{1+t}{2} a+\frac{1-t}{2} b$, we get

$$
\begin{aligned}
\frac{1}{h\left(\frac{1}{2}\right)} F\left(\frac{a+b}{2}\right) \supseteq & F\left(\frac{1-t}{2} a+\frac{1+t}{2} b\right)+F\left(\frac{1+t}{2} a+\frac{1-t}{2} b\right) \\
& +\frac{1}{h\left(\frac{1}{2}\right)} H\left(\frac{1-t}{2} a+\frac{1+t}{2} b, \frac{1+t}{2} a+\frac{1-t}{2} b\right) .
\end{aligned}
$$

Multiplying by $\frac{\varphi\left(\frac{(b-a)}{2} t\right)}{t}$ both sides of inequality (5.51) and integrating the resultant one with respect to $t$ over $[0,1]$, we obtain

$$
\begin{aligned}
& \frac{1}{h\left(\frac{1}{2}\right)} F\left(\frac{a+b}{2}\right) \int_{0}^{1} \frac{\varphi\left(\frac{(b-a)}{2} t\right)}{t} d t \\
& \supseteq(I R) \int_{0}^{1} \frac{\varphi\left(\frac{(b-a)}{2} t\right)}{t} F\left(\frac{1-t}{2} a+\frac{1+t}{2} b\right) d t \\
& \quad+(I R) \int_{0}^{1} \frac{\varphi\left(\frac{(b-a)}{2} t\right)}{t} F\left(\frac{1+t}{2} a+\frac{1-t}{2} b\right) d t \\
& \quad+\frac{1}{h\left(\frac{1}{2}\right)} \int_{0}^{1} \frac{\varphi\left(\frac{(b-a)}{2} t\right)}{t} H\left(\frac{1-t}{2} a+\frac{1+t}{2} b, \frac{1+t}{2} a+\frac{1-t}{2} b\right) d t .
\end{aligned}
$$

By using Theorem 1 in Eq. (5.52), we have

$$
\begin{aligned}
\text { (IR) } & \int_{0}^{1} \frac{\varphi\left(\frac{(b-a)}{2} t\right)}{t} F\left(\frac{1-t}{2} a+\frac{1+t}{2} b\right) d t \\
= & {\left[(R) \int_{0}^{1} \frac{\varphi\left(\frac{(b-a)}{2} t\right)}{t} \underline{F}\left(\frac{1-t}{2} a+\frac{1+t}{2} b\right) d t\right.} \\
& \left.(R) \int_{0}^{1} \frac{\varphi\left(\frac{(b-a)}{2} t\right)}{t} \bar{F}\left(\frac{1-t}{2} a+\frac{1+t}{2} b\right) d t\right] \\
= & {\left[(R) \int_{\frac{a+b}{2}}^{b} \frac{\varphi\left(u-\frac{a+b}{2}\right)}{u-\frac{a+b}{2}} \underline{F}(u) d u,(R) \int_{\frac{a+b}{2}}^{b} \frac{\varphi\left(u-\frac{a+b}{2}\right)}{u-\frac{a+b}{2}} \bar{F}(u) d u\right] } \\
= & {\left[b_{b} I_{\varphi} \underline{F}\left(\frac{a+b}{2}\right),{ }_{b-} I_{\varphi} \bar{F}\left(\frac{a+b}{2}\right)\right] } \\
= & { }_{b-} \mathfrak{I}_{\varphi} F\left(\frac{a+b}{2}\right) .
\end{aligned}
$$

Similarly, we get

$$
\begin{aligned}
\text { (IR) } \int_{0}^{1} \frac{\varphi\left(\frac{(b-a)}{2} t\right)}{t} F\left(\frac{1+t}{2} a+\frac{1-t}{2} b\right) d t & =\left[{ }_{a+} I_{\varphi} F\left(\frac{a+b}{2}\right),{ }_{a+} I_{\varphi} \bar{F}\left(\frac{a+b}{2}\right)\right] \\
& ={ }_{a+} \mathfrak{I}_{\varphi} F\left(\frac{a+b}{2}\right) .
\end{aligned}
$$


Hence, we proved the first inequality. To prove the second inequality of (5.50), first we note that, since $F$ is an interval-valued approximately $h$-convex function, we have

$$
F\left(\frac{1+t}{2} a+\frac{1-t}{2} b\right) \supseteq h\left(\frac{1+t}{2}\right) F(a)+h\left(\frac{1-t}{2}\right) F(b)+H(a, b)
$$

and

$$
F\left(\frac{1-t}{2} a+\frac{1+t}{2} b\right) \supseteq h\left(\frac{1-t}{2}\right) F(a)+h\left(\frac{1+t}{2}\right) F(b)+H(a, b) .
$$

Adding (5.53) and (5.54), we have

$$
\begin{aligned}
& F\left(\frac{1+t}{2} a+\frac{1-t}{2} b\right)+F\left(\frac{1-t}{2} a+\frac{1+t}{2} b\right) \\
& \quad \supseteq[F(a)+F(b)]\left[h\left(\frac{1-t}{2}\right)+h\left(\frac{1+t}{2}\right)\right]+2 H(a, b) .
\end{aligned}
$$

Multiplying by $\frac{\varphi\left(\frac{(b-a)}{2} t\right)}{t}$ both sides of inequality (5.55) and integrating the resultant one with respect to $t$ over $[0,1]$, we obtain

$$
\begin{aligned}
& \text { (IR) } \int_{0}^{1} \frac{\varphi\left(\frac{(b-a)}{2} t\right)}{t} F\left(\frac{1+t}{2} a+\frac{1-t}{2} b\right) d t+(I R) \int_{0}^{1} \frac{\varphi\left(\frac{(b-a)}{2} t\right)}{t} F\left(\frac{1-t}{2} a+\frac{1+t}{2} b\right) d t \\
& \supseteq[F(a)+F(b)] \int_{0}^{1} \frac{\varphi\left(\frac{(b-a)}{2} t\right)}{t}\left[h\left(\frac{1-t}{2}\right)+h\left(\frac{1+t}{2}\right)\right] d t+2 H(a, b) \int_{0}^{1} \frac{\varphi\left(\frac{(b-a)}{2} t\right)}{t} d t .
\end{aligned}
$$

This completes the proof.

Corollary 14 If we choose $\varphi(t)=t$ in Theorem 12, then we have the following inequalities:

$$
\begin{aligned}
& \frac{1}{2 h\left(\frac{1}{2}\right)} F\left(\frac{a+b}{2}\right)-\frac{1}{(b-a) h\left(\frac{1}{2}\right)} \int_{\frac{a+b}{2}}^{b} H(x, a+b-x) d x \\
& \supseteq \frac{1}{b-a}(I R) \int_{a}^{b} F(x) d x \supseteq \frac{[F(a)+F(b)]}{2} \int_{0}^{1}\left[h\left(\frac{1-t}{2}\right)+h\left(\frac{1+t}{2}\right)\right] d t+H(a, b) .
\end{aligned}
$$

Corollary 15 Taking $\varphi(t)=\frac{t^{\alpha}}{\Gamma(\alpha)}$ in Theorem 12, then we have the following inequalities for the interval-valued fractional operators:

$$
\begin{aligned}
& \frac{1}{2 h\left(\frac{1}{2}\right)} F\left(\frac{a+b}{2}\right)-\frac{\alpha 2^{\alpha-1}}{(b-a)^{\alpha} h\left(\frac{1}{2}\right)} \int_{\frac{a+b}{2}}^{b}\left(x-\frac{a+b}{2}\right)^{\alpha-1} H(x, a+b-x) d x \\
& \supseteq \frac{2^{\alpha-1} \Gamma(\alpha+1)}{(b-a)^{\alpha}}\left[J_{a+}^{\alpha} F\left(\frac{a+b}{2}\right)+J_{b-}^{\alpha} F\left(\frac{a+b}{2}\right)\right] \\
& \supseteq \frac{\alpha[F(a)+F(b)]}{2} \int_{0}^{1} t^{\alpha-1}\left[h\left(\frac{1-t}{2}\right)+h\left(\frac{1+t}{2}\right)\right] d t+H(a, b) .
\end{aligned}
$$

Theorem 13 If $F, G:[a, b] \rightarrow \mathbb{R}_{\mathcal{I}}^{+}$are two interval-valued approximately $h$-convex functions such that $F(t)=[\underline{F}(t), \bar{F}(t)]$ and $G(t)=[\underline{G}(t), \bar{G}(t)]$, then we have the following inequal- 
ity for the generalized fractional integrals:

$$
\begin{aligned}
& {\left[a^{+} \Im_{\varphi} F\left(\frac{a+b}{2}\right) G\left(\frac{a+b}{2}\right)+b^{-} \Im_{\varphi} F\left(\frac{a+b}{2}\right) G\left(\frac{a+b}{2}\right)\right]} \\
& \supseteq K_{7} M(a, b)+K_{8} N(a, b)+K_{9} H(a, b) P(a, b)+2 \psi(1) H^{2}(a, b),
\end{aligned}
$$

where $M(a, b)$ and $N(a, b)$ are defined in Theorem 3 and

$$
\begin{aligned}
& K_{7}=\int_{0}^{1} \frac{\varphi\left(\frac{(b-a)}{2} t\right)}{t}\left[h^{2}\left(\frac{1+t}{2}\right)+h^{2}\left(\frac{1-t}{2}\right)\right] d t, \\
& K_{8}=2 \int_{0}^{1} \frac{\varphi\left(\frac{(b-a)}{2} t\right)}{t} h\left(\frac{1+t}{2}\right) h\left(\frac{1-t}{2}\right) d t, \\
& K_{9}=\int_{0}^{1} \frac{\varphi\left(\frac{(b-a)}{2} t\right)}{t}\left[h\left(\frac{1+t}{2}\right)+h\left(\frac{1-t}{2}\right)\right] d t .
\end{aligned}
$$

Proof Since $F$ and $G$ are two interval-valued approximately $h$-convex functions,

$$
F\left(\frac{1-t}{2} a+\frac{1+t}{2} b\right) \supseteq h\left(\frac{1-t}{2}\right) F(a)+h\left(\frac{1+t}{2}\right) F(b)+H(a, b)
$$

and

$$
G\left(\frac{1-t}{2} a+\frac{1+t}{2} b\right) \supseteq h\left(\frac{1-t}{2}\right) G(a)+h\left(\frac{1+t}{2}\right) G(b)+H(a, b) .
$$

Multiplying (5.59) and (5.60), we have

$$
\begin{aligned}
F( & \left.\frac{1-t}{2} a+\frac{1+t}{2} b\right) G\left(\frac{1-t}{2} a+\frac{1+t}{2} b\right) \\
\supseteq & h^{2}\left(\frac{1-t}{2}\right) F(a) G(a)+h^{2}\left(\frac{1+t}{2}\right) F(b) G(b) \\
& +h\left(\frac{1-t}{2}\right) h\left(\frac{1+t}{2}\right)[F(a) G(b)+F(b) G(a)] \\
& +h\left(\frac{1+t}{2}\right) H(a, b)[F(b)+G(b)]+h\left(\frac{1-t}{2}\right) H(a, b)[F(a)+G(a)]+H^{2}(a, b) .
\end{aligned}
$$

Similarly, we get

$$
\begin{aligned}
F( & \left.\frac{1+t}{2} a+\frac{1-t}{2} b\right) G\left(\frac{1+t}{2} a+\frac{1-t}{2} b\right) \\
\supseteq & h^{2}\left(\frac{1+t}{2}\right) F(a) G(a)+h^{2}\left(\frac{1-t}{2}\right) F(b) G(b) \\
& +h\left(\frac{1+t}{2}\right) h\left(\frac{1-t}{2}\right)[F(a) G(b)+F(b) G(a)] \\
& +h\left(\frac{1-t}{2}\right) H(a, b)[F(b)+G(b)]+h\left(\frac{1+t}{2}\right) H(a, b)[F(a)+G(a)]+H^{2}(a, b) .
\end{aligned}
$$


Adding (5.61) and (5.62), we obtain the following relation:

$$
\begin{aligned}
F( & \left.\frac{1-t}{2} a+\frac{1+t}{2} b\right) G\left(\frac{1-t}{2} a+\frac{1+t}{2} b\right) \\
& +F\left(\frac{1+t}{2} a+\frac{1-t}{2} b\right) G\left(\frac{1+t}{2} a+\frac{1-t}{2} b\right) \\
\supseteq & h^{2}\left(\frac{1-t}{2}\right)[F(a) G(a)+F(b) G(b)] \\
& +h^{2}\left(\frac{1+t}{2}\right)[F(a) G(a)+F(b) G(b)] \\
& +2 h\left(\frac{1+t}{2}\right) h\left(\frac{1-t}{2}\right)[F(a) G(b)+F(b) G(a)] \\
& +H(a, b)\left[h\left(\frac{1+t}{2}\right)+h\left(\frac{1-t}{2}\right)\right][F(a)+F(b)+G(a)+G(b)]+2 H^{2}(a, b) \\
= & {\left[h^{2}\left(\frac{1-t}{2}\right)+h^{2}\left(\frac{1+t}{2}\right)\right] M(a, b)+2 h\left(\frac{1+t}{2}\right) h\left(\frac{1-t}{2}\right) N(a, b) } \\
& +H(a, b) P(a, b)\left[h\left(\frac{1+t}{2}\right)+h\left(\frac{1-t}{2}\right)\right]+2 H^{2}(a, b) .
\end{aligned}
$$

Multiplying by $\frac{\varphi\left(\frac{(b-a)}{2} t\right)}{t}$ both sides of inequality (5.63) and integrating the resultant one with respect to $t$ over $[0,1]$, we have

$$
\begin{aligned}
& \text { (IR) } \int_{0}^{1} \frac{\varphi\left(\frac{(b-a)}{2} t\right)}{t} F\left(\frac{1-t}{2} a+\frac{1+t}{2} b\right) G\left(\frac{1-t}{2} a+\frac{1+t}{2} b\right) d t \\
& \quad+(I R) \int_{0}^{1} \frac{\varphi\left(\frac{(b-a)}{2} t\right)}{t} F\left(\frac{1+t}{2} a+\frac{1-t}{2} b\right) G\left(\frac{1+t}{2} a+\frac{1-t}{2} b\right) d t \\
& \supseteq M(a, b) \int_{0}^{1} \frac{\varphi\left(\frac{(b-a)}{2} t\right)}{t}\left[h^{2}\left(\frac{1-t}{2}\right)+h^{2}\left(\frac{1+t}{2}\right)\right] d t \\
& +2 N(a, b) \int_{0}^{1} \frac{\varphi\left(\frac{(b-a)}{2} t\right)}{t} h\left(\frac{1+t}{2}\right) h\left(\frac{1-t}{2}\right) d t \\
& +H(a, b) P(a, b) \int_{0}^{1} \frac{\varphi\left(\frac{(b-a)}{2} t\right)}{t}\left[h\left(\frac{1+t}{2}\right)+h\left(\frac{1-t}{2}\right)\right] d t \\
& +2 H^{2}(a, b) \int_{0}^{1} \frac{\varphi\left(\frac{(b-a)}{2} t\right)}{t} d t .
\end{aligned}
$$

By using Theorem 1 in Eq. (5.64), we obtain our required inequality.

Corollary 16 If we choose $\varphi(t)=t$ in Theorem 13, then we get the following inequality:

$$
\begin{aligned}
& \frac{2}{b-a}(I R) \int_{a}^{b} F(x) G(x) d x \\
& \supseteq M(a, b) \int_{0}^{1}\left[h^{2}\left(\frac{1+t}{2}\right)+h^{2}\left(\frac{1-t}{2}\right)\right] d t+2 N(a, b) \int_{0}^{1} h\left(\frac{1+t}{2}\right) h\left(\frac{1+t}{2}\right) d t \\
& \quad+H(a, b) P(a, b) \int_{0}^{1}\left[h\left(\frac{1+t}{2}\right)+h\left(\frac{1-t}{2}\right)\right] d t+2 H^{2}(a, b) .
\end{aligned}
$$


Corollary 17 Taking $\varphi(t)=\frac{t^{\alpha}}{\Gamma(\alpha)}$ in Theorem 13, then we have the following inequality for the interval-valued fractional operators:

$$
\begin{aligned}
& \frac{2^{\alpha} \Gamma(\alpha+1)}{(b-a)^{\alpha}}\left[J_{\left(\frac{a+b}{2}\right)+}^{\alpha} F(b) G(b)+J_{\left(\frac{a+b}{2}\right)-}^{\alpha} F(a) G(a)\right] \\
& \supseteq \alpha\left\{M(a, b) \int_{0}^{1} t^{\alpha-1}\left[h^{2}\left(\frac{1+t}{2}\right)+h^{2}\left(\frac{1-t}{2}\right)\right] d t\right. \\
& \quad+2 N(a, b) \int_{0}^{1} t^{\alpha-1} h\left(\frac{1+t}{2}\right) h\left(\frac{1-t}{2}\right) d t \\
& \left.\quad+H(a, b) P(a, b) \int_{0}^{1} t^{\alpha-1}\left[h\left(\frac{1+t}{2}\right)+h\left(\frac{1-t}{2}\right)\right] d t\right\}+2 H^{2}(a, b) .
\end{aligned}
$$

Theorem 14 If $F, G:[a, b] \rightarrow \mathbb{R}_{\mathcal{I}}^{+}$are two interval-valued approximately h-convex functions such that $F(t)=[\underline{F}(t), \bar{F}(t)]$ and $G(t)=[\underline{G}(t), \bar{G}(t)]$, then we have the following inequality for the generalized fractional integrals:

$$
\begin{aligned}
\frac{1}{h^{2}\left(\frac{1}{2}\right)} & F\left(\frac{a+b}{2}\right) G\left(\frac{a+b}{2}\right) \\
& -\frac{1}{h\left(\frac{1}{2}\right) \psi(1)}\left[(I R) \int_{\frac{a+b}{2}}^{b} \frac{\varphi\left(x-\frac{a+b}{2}\right)}{x-\frac{a+b}{2}}[F(x)+G(x)] H(x, a+b-x) d x\right. \\
& \left.+(I R) \int_{a}^{\frac{a+b}{2}} \frac{\varphi\left(\frac{a+b}{2}-x\right)}{\frac{a+b}{2}-x}[F(x)+G(x)] H(a+b-x, x) d x\right] \\
\supseteq & \frac{1}{\psi(1)}\left[a^{+} \Im_{\varphi} F\left(\frac{a+b}{2}\right) G\left(\frac{a+b}{2}\right)+{ }_{b^{-}} \Im_{\varphi} F\left(\frac{a+b}{2}\right) G\left(\frac{a+b}{2}\right)\right] \\
& +\frac{1}{\psi(1) h^{2}\left(\frac{1}{2}\right)} \int_{\frac{a+b}{2}}^{b} \frac{\varphi\left(x-\frac{a+b}{2}\right)}{x-\frac{a+b}{2}} H^{2}(x, a+b-x) d x \\
& +\frac{1}{\psi(1)}\left[K_{8} M(a, b)+K_{7} N(a, b)+K_{9} P(a, b) H(a, b)\right]+2 H^{2}(a, b),
\end{aligned}
$$

where $M(a, b), N(a, b)$ and $K_{7}, K_{8}, K_{9}$ are defined in Theorem 3 and Theorem 13, respectively.

Proof Since $F$ and $G$ are two interval-valued approximately $h$-convex functions on $[a, b]$, we have

$$
\frac{1}{h\left(\frac{1}{2}\right)} F\left(\frac{x+y}{2}\right) \supseteq F(x)+F(y)+\frac{1}{h\left(\frac{1}{2}\right)} H(x, y) .
$$

For $x=\frac{1-t}{2} a+\frac{1+t}{2} b$ and $y=\frac{1+t}{2} a+\frac{1-t}{2} b$, we obtain

$$
\begin{aligned}
\frac{1}{h\left(\frac{1}{2}\right)} F\left(\frac{a+b}{2}\right) \supseteq & F\left(\frac{1-t}{2} a+\frac{1+t}{2} b\right)+F\left(\frac{1+t}{2} a+\frac{1-t}{2} b\right) \\
& +\frac{1}{h\left(\frac{1}{2}\right)} H\left(\frac{1-t}{2} a+\frac{1+t}{2} b, \frac{1+t}{2} a+\frac{1-t}{2} b\right) .
\end{aligned}
$$


Similarly, we get

$$
\begin{aligned}
\frac{1}{h\left(\frac{1}{2}\right)} G\left(\frac{a+b}{2}\right) \supseteq & G\left(\frac{1-t}{2} a+\frac{1+t}{2} b\right)+G\left(\frac{1+t}{2} a+\frac{1-t}{2} b\right) \\
& +\frac{1}{h\left(\frac{1}{2}\right)} H\left(\frac{1-t}{2} a+\frac{1+t}{2} b, \frac{1+t}{2} a+\frac{1-t}{2} b\right) .
\end{aligned}
$$

Multiplying the inequalities (5.69) and (5.70), we obtain

$$
\begin{aligned}
& \frac{1}{h^{2}\left(\frac{1}{2}\right)} F\left(\frac{a+b}{2}\right) G\left(\frac{a+b}{2}\right) \\
& \supseteq F\left(\frac{1-t}{2} a+\frac{1+t}{2} b\right) G\left(\frac{1-t}{2} a+\frac{1+t}{2} b\right) \\
& +F\left(\frac{1+t}{2} a+\frac{1-t}{2} b\right) G\left(\frac{1+t}{2} a+\frac{1-t}{2} b\right) \\
& +F\left(\frac{1-t}{2} a+\frac{1+t}{2} b\right) G\left(\frac{1+t}{2} a+\frac{1-t}{2} b\right) \\
& +F\left(\frac{1+t}{2} a+\frac{1-t}{2} b\right) G\left(\frac{1-t}{2} a+\frac{1+t}{2} b\right) \\
& +\frac{1}{h^{2}\left(\frac{1}{2}\right)} H^{2}\left(\frac{1-t}{2} a+\frac{1+t}{2} b, \frac{1+t}{2} a+\frac{1-t}{2} b\right) \\
& +\frac{1}{h\left(\frac{1}{2}\right)} F\left(\frac{1-t}{2} a+\frac{1+t}{2} b\right) H\left(\frac{1-t}{2} a+\frac{1+t}{2} b, \frac{1+t}{2} a+\frac{1-t}{2} b\right) \\
& +\frac{1}{h\left(\frac{1}{2}\right)} F\left(\frac{1+t}{2} a+\frac{1-t}{2} b\right) H\left(\frac{1-t}{2} a+\frac{1+t}{2} b, \frac{1+t}{2} a+\frac{1-t}{2} b\right) \\
& +\frac{1}{h\left(\frac{1}{2}\right)} G\left(\frac{1-t}{2} a+\frac{1+t}{2} b\right) H\left(\frac{1-t}{2} a+\frac{1+t}{2} b, \frac{1+t}{2} a+\frac{1-t}{2} b\right) \\
& +\frac{1}{h\left(\frac{1}{2}\right)} G\left(\frac{1+t}{2} a+\frac{1-t}{2} b\right) H\left(\frac{1-t}{2} a+\frac{1+t}{2} b, \frac{1+t}{2} a+\frac{1-t}{2} b\right) \\
& \supseteq F\left(\frac{1-t}{2} a+\frac{1+t}{2} b\right) G\left(\frac{1-t}{2} a+\frac{1+t}{2} b\right) \\
& +F\left(\frac{1+t}{2} a+\frac{1-t}{2} b\right) G\left(\frac{1+t}{2} a+\frac{1-t}{2} b\right) \\
& +\left[h\left(\frac{1-t}{2}\right) F(a)+h\left(\frac{1+t}{2}\right) F(b)+H(a, b)\right] \\
& \times\left[h\left(\frac{1+t}{2}\right) G(a)+h\left(\frac{1-t}{2}\right) G(b)+H(a, b)\right] \\
& +\left[h\left(\frac{1+t}{2}\right) F(a)+h\left(\frac{1-t}{2}\right) F(b)+H(a, b)\right] \\
& \times\left[h\left(\frac{1-t}{2}\right) G(a)+h\left(\frac{1+t}{2}\right) G(b)+H(a, b)\right] \\
& +\frac{1}{h^{2}\left(\frac{1}{2}\right)} H^{2}\left(\frac{1-t}{2} a+\frac{1+t}{2} b, \frac{1+t}{2} a+\frac{1-t}{2} b\right)
\end{aligned}
$$




$$
\begin{aligned}
& +\frac{1}{h\left(\frac{1}{2}\right)} F\left(\frac{1-t}{2} a+\frac{1+t}{2} b\right) H\left(\frac{1-t}{2} a+\frac{1+t}{2} b, \frac{1+t}{2} a+\frac{1-t}{2} b\right) \\
& +\frac{1}{h\left(\frac{1}{2}\right)} F\left(\frac{1+t}{2} a+\frac{1-t}{2} b\right) H\left(\frac{1-t}{2} a+\frac{1+t}{2} b, \frac{1+t}{2} a+\frac{1-t}{2} b\right) \\
& +\frac{1}{h\left(\frac{1}{2}\right)} G\left(\frac{1-t}{2} a+\frac{1+t}{2} b\right) H\left(\frac{1-t}{2} a+\frac{1+t}{2} b, \frac{1+t}{2} a+\frac{1-t}{2} b\right) \\
& +\frac{1}{h\left(\frac{1}{2}\right)} G\left(\frac{1+t}{2} a+\frac{1-t}{2} b\right) H\left(\frac{1-t}{2} a+\frac{1+t}{2} b, \frac{1+t}{2} a+\frac{1-t}{2} b\right) \\
& +F\left(\frac{1-t}{2} a+\frac{1+t}{2} b\right) G\left(\frac{1-t}{2} a+\frac{1+t}{2} b\right) \\
& +F\left(\frac{1+t}{2} a+\frac{1-t}{2} b\right) G\left(\frac{1+t}{2} a+\frac{1-t}{2} b\right) \\
& +\frac{1}{h\left(\frac{1}{2}\right)} G\left(\frac{1+t}{2} a+\frac{1-t}{2} b\right) H\left(\frac{1-t}{2} a+\frac{1+t}{2} b, \frac{1+t}{2} a+\frac{1-t}{2} b\right)+2 H^{2}(a, b) . \\
& + \\
& +
\end{aligned}
$$

Multiplying by $\frac{\varphi\left(\frac{(b-a)}{2} t\right)}{t}$ both sides of inequality (5.71) and integrating the resultant one with respect to $t$ over $[0,1]$, we obtain our result (5.67).

Corollary 18 Taking $\varphi(t)=t$ in Theorem 14, then we have the following inequality:

$$
\begin{aligned}
& \frac{1}{h^{2}\left(\frac{1}{2}\right)} F\left(\frac{a+b}{2}\right) G\left(\frac{a+b}{2}\right) \\
& \quad-\frac{2}{(b-a) h\left(\frac{1}{2}\right)}\left[(I R) \int_{\frac{a+b}{2}}^{b}[F(x)+G(x)] H(x, a+b-x) d x\right. \\
& \left.\quad+(I R) \int_{a}^{\frac{a+b}{2}}[F(x)+G(x)] H(a+b-x, x) d x\right] \\
& \supseteq \frac{2}{b-a}(I R) \int_{a}^{b} F(x) G(x) d x+\frac{2}{(b-a) h^{2}\left(\frac{1}{2}\right)} \int_{\frac{a+b}{2}}^{b} H^{2}(x, a+b-x) d x
\end{aligned}
$$




$$
\begin{aligned}
& +2 M(a, b) \int_{0}^{1} h\left(\frac{1+t}{2}\right) h\left(\frac{1-t}{2}\right) d t+N(a, b) \int_{0}^{1}\left[h^{2}\left(\frac{1+t}{2}\right)+h^{2}\left(\frac{1-t}{2}\right)\right] d t \\
& +P(a, b) H(a, b) \int_{0}^{1}\left[h\left(\frac{1+t}{2}\right)+h\left(\frac{1-t}{2}\right)\right] d t+2 H^{2}(a, b) .
\end{aligned}
$$

Corollary 19 Taking $\varphi(t)=\frac{t^{\alpha}}{\Gamma(\alpha)}$ in Theorem 14, then we have the following inequality for the interval-valued fractional operators:

$$
\begin{aligned}
\frac{1}{h^{2}\left(\frac{1}{2}\right)} & F\left(\frac{a+b}{2}\right) G\left(\frac{a+b}{2}\right) \\
& -\frac{2^{\alpha} \alpha}{(b-a)^{\alpha} h\left(\frac{1}{2}\right)}\left[(I R) \int_{\frac{a+b}{2}}^{b}\left(x-\frac{a+b}{2}\right)^{\alpha-1}[F(x)+G(x)] H(x, a+b-x) d x\right. \\
& \left.+(I R) \int_{a}^{\frac{a+b}{2}}\left(\frac{a+b}{2}-x\right)^{\alpha-1}[F(x)+G(x)] H(a+b-x, y) d x\right] \\
\supseteq & \frac{2^{\alpha} \Gamma(\alpha+1)}{(b-a)^{\alpha}}\left[J_{\left(\frac{a+b}{2}\right)+}^{\alpha} F(b) G(b)+J_{\left(\frac{a+b}{2}\right)-}^{\alpha} F(a) G(a)\right] \\
& +\frac{\alpha 2^{\alpha}}{(b-a)^{\alpha} h^{2}\left(\frac{1}{2}\right)} \int_{\frac{a+b}{2}}^{b}\left(x-\frac{a+b}{2}\right)^{\alpha-1} H^{2}(x, a+b-x) d x \\
& +\alpha\left\{2 M(a, b) \int_{0}^{1} t^{\alpha-1} h\left(\frac{1+t}{2}\right) h\left(\frac{1-t}{2}\right) d t\right. \\
& +N(a, b) \int_{0}^{1} t^{\alpha-1}\left[h^{2}\left(\frac{1+t}{2}\right)+h^{2}\left(\frac{1-t}{2}\right)\right] d t \\
& \left.+P(a, b) H(a, b) \int_{0}^{1} t^{\alpha-1}\left[h\left(\frac{1+t}{2}\right)+h\left(\frac{1-t}{2}\right)\right] d t\right\}+2 H^{2}(a, b) .
\end{aligned}
$$

\section{Some special cases}

In this section, we discuss some special cases from our main results.

From Theorem 6, we have the following result.

Corollary 20 If $F:[a, b] \rightarrow \mathbb{R}_{\mathcal{I}}^{+}$is interval-valued $\gamma$-approximately h-convex function, then

$$
\begin{aligned}
& \frac{1}{2 h\left(\frac{1}{2}\right)} F\left(\frac{a+b}{2}\right)-\frac{\epsilon}{2 h\left(\frac{1}{2}\right) \Lambda(1)} \int_{a}^{b} \frac{\varphi(b-x)}{b-x}(\|2 x-(a+b)\|)^{\gamma} d x \\
& \supseteq \frac{1}{2 \Lambda(1)}\left[a+\Im_{\varphi} F(b)+{ }_{b-} \mathfrak{I}_{\varphi} F(a)\right] \\
& \supseteq \frac{F(a)+F(b)}{2 \Lambda(1)} \int_{0}^{1}[h(t)+h(1-t)] \frac{\varphi((b-a) t)}{t} d t+\epsilon(\|b-a\|)^{\gamma} .
\end{aligned}
$$

Corollary 21 If $F, G:[a, b] \rightarrow \mathbb{R}_{\mathcal{I}}^{+}$are two interval-valued $\gamma$-approximately $h$-convex functions, then

$$
\begin{aligned}
& \frac{1}{2}\left[{ }_{a+} \Im_{\varphi} F(b) G(b)+{ }_{b-} \Im_{\varphi} F(a) G(a)\right] \\
& \quad \supseteq K_{1} M(a, b)+K_{2} N(a, b)+K_{3} P(a, b) \epsilon(\|b-a\|)^{\gamma}+\Lambda(1) \epsilon^{2}(\|b-a\|)^{2 \gamma} .
\end{aligned}
$$


Corollary 22 If $F, G:[a, b] \rightarrow \mathbb{R}_{\mathcal{I}}^{+}$are two interval-valued $\epsilon$-approximately h-convex functions, then

$$
\begin{aligned}
& \frac{1}{2}\left[{ }_{a+} \Im_{\varphi} F(b) G(b)+{ }_{b-} \Im_{\varphi} F(a) G(a)\right] \\
& \quad \supseteq K_{1} M(a, b)+K_{2} N(a, b)+K_{3} P(a, b) \epsilon(\|b-a\|)+\Lambda(1) \epsilon^{2}(\|b-a\|)^{2} .
\end{aligned}
$$

From Theorem 8, we obtain the following result.

Corollary 23 If $F, G:[a, b] \rightarrow \mathbb{R}_{\mathcal{I}}^{+}$are two interval-valued $\gamma$-approximately h-convex functions, then

$$
\begin{aligned}
& \frac{1}{2 h^{2}\left(\frac{1}{2}\right)} F\left(\frac{a+b}{2}\right) G\left(\frac{a+b}{2}\right) \\
& \quad-\frac{\epsilon}{2 \Lambda(1) h\left(\frac{1}{2}\right)}\left[(I R) \int_{a}^{b} \frac{\varphi(b-x)}{b-x}(F(x)+G(x))(\|2 x-(a+b)\|)^{\gamma} d x\right. \\
& \left.\quad+(I R) \int_{a}^{b} \frac{\varphi(x-a)}{x-a}(F(x)+G(x))(\|a+b-2 x\|)^{\gamma} d x\right] \\
& \supseteq \frac{1}{2 \Lambda(1)}\left[{ }_{a+} \Im_{\varphi} F(b) G(b)+{ }_{b-} \Im_{\varphi} F(a) G(a)\right] \\
& \quad+\frac{\epsilon^{2}}{2 \Lambda(1) h^{2}\left(\frac{1}{2}\right)} \int_{a}^{b} \frac{\varphi(b-x)}{b-x}(\|2 x-(a+b)\|)^{2 \gamma} d x \\
& \quad+\frac{1}{\Lambda(1)}\left[K_{2} M(a, b)+K_{1} N(a, b)+K_{3} P(a, b) \epsilon(\|b-a\|)^{\gamma}\right]+\epsilon^{2}(\|b-a\|)^{2 \gamma} .
\end{aligned}
$$

Corollary 24 If $F, G:[a, b] \rightarrow \mathbb{R}_{\mathcal{I}}^{+}$are two interval-valued $\epsilon$-approximately h-convex functions, then

$$
\begin{aligned}
& \frac{1}{2 h^{2}\left(\frac{1}{2}\right)} F\left(\frac{a+b}{2}\right) G\left(\frac{a+b}{2}\right) \\
& \quad-\frac{\epsilon}{2 \Lambda(1) h\left(\frac{1}{2}\right)}\left[(I R) \int_{a}^{b} \frac{\varphi(b-x)}{b-x}(F(x)+G(x))(\|2 x-(a+b)\|) d x\right. \\
& \left.\quad+(I R) \int_{a}^{b} \frac{\varphi(x-a)}{x-a}(F(x)+G(x))(\|a+b-2 x\|) d x\right] \\
& \supseteq \frac{1}{2 \Lambda(1)}\left[{ }_{a+} \Im_{\varphi} F(b) G(b)+{ }_{b-} \Im_{\varphi} F(a) G(a)\right] \\
& \quad+\frac{\epsilon^{2}}{2 \Lambda(1) h^{2}\left(\frac{1}{2}\right)} \int_{a}^{b} \frac{\varphi(b-x)}{b-x}(\|2 x-(a+b)\|)^{2} d x \\
& \quad+\frac{1}{\Lambda(1)}\left[K_{2} M(a, b)+K_{1} N(a, b)+K_{3} P(a, b) \epsilon(\|b-a\|)\right]+\epsilon^{2}(\|b-a\|)^{2} .
\end{aligned}
$$

From Theorem 9, we have the following result. 
Corollary 25 If $F:[a, b] \rightarrow \mathbb{R}_{\mathcal{I}}^{+}$is interval-valued $\gamma$-approximately h-convex function, then

$$
\begin{aligned}
& \frac{1}{2 h\left(\frac{1}{2}\right)} F\left(\frac{a+b}{2}\right)-\frac{\epsilon}{2 \psi(1) h\left(\frac{1}{2}\right)} \int_{\frac{a+b}{2}}^{b} \frac{\varphi(b-x)}{b-x}(\|2 x-(a+b)\|)^{\gamma} d x \\
& \supseteq \frac{1}{2 \psi(1)}\left[\left(\frac{a+b}{2}\right)^{+} \Im_{\varphi} F(b)+\left(\frac{a+b}{2}\right)^{-} \Im_{\varphi} F(a)\right] \\
& \supseteq \frac{[F(a)+F(b)]}{2 \psi(1)} \int_{0}^{1} \frac{\varphi\left(\frac{(b-a)}{2} t\right)}{t}\left[h\left(\frac{2-t}{2}\right)+h\left(\frac{t}{2}\right)\right] d t+\epsilon(\|b-a\|)^{\gamma} .
\end{aligned}
$$

Corollary 26 If $F:[a, b] \rightarrow \mathbb{R}_{\mathcal{I}}^{+}$is interval-valued $\epsilon$-approximately h-convex function, then

$$
\begin{aligned}
& \frac{1}{2 h\left(\frac{1}{2}\right)} F\left(\frac{a+b}{2}\right)-\frac{\epsilon}{2 \psi(1) h\left(\frac{1}{2}\right)} \int_{\frac{a+b}{2}}^{b} \frac{\varphi(b-x)}{b-x}(\|2 x-(a+b)\|) d x \\
& \supseteq \frac{1}{2 \psi(1)}\left[\left(\frac{a+b}{2}\right)^{+} \mathfrak{I}_{\varphi} F(b)+{ }_{\left(\frac{a+b}{2}\right)^{-}} \mathfrak{I}_{\varphi} F(a)\right] \\
& \supseteq \frac{[F(a)+F(b)]}{2 \psi(1)} \int_{0}^{1} \frac{\varphi\left(\frac{(b-a)}{2} t\right)}{t}\left[h\left(\frac{2-t}{2}\right)+h\left(\frac{t}{2}\right)\right] d t+\epsilon(\|b-a\|) .
\end{aligned}
$$

From Theorem 10, we get

Corollary 27 If $F, G:[a, b] \rightarrow \mathbb{R}_{\mathcal{I}}^{+}$are two interval-valued $\gamma$-approximately h-convex functions, then

$$
\begin{aligned}
& {\left[{ }_{\left(\frac{a+b}{2}\right)^{+}} \Im_{\varphi} F(b) G(b)+_{\left(\frac{a+b}{2}\right)^{-}} \Im_{\varphi} F(a) G(a)\right]} \\
& \quad \supseteq K_{4} M(a, b)+K_{5} N(a, b)+\epsilon^{2}(\|b-a\|)^{\gamma} P(a, b) K_{6}+2 \psi(1) \epsilon^{2}(\|b-a\|)^{2 \gamma} .
\end{aligned}
$$

Corollary 28 If $F, G:[a, b] \rightarrow \mathbb{R}_{\mathcal{I}}^{+}$are two interval-valued $\epsilon$-approximately h-convex functions, then

$$
\begin{aligned}
& {\left[\left(\frac{a+b}{2}\right)^{+} \Im_{\varphi} F(b) G(b)+{ }_{\left(\frac{a+b}{2}\right)^{-}} \Im_{\varphi} F(a) G(a)\right]} \\
& \quad \supseteq K_{4} M(a, b)+K_{5} N(a, b)+\epsilon^{2}(\|b-a\|) P(a, b) K_{6}+2 \psi(1) \epsilon^{2}(\|b-a\|)^{2} .
\end{aligned}
$$

From Theorem 11, we obtain the following result.

Corollary 29 If $F, G:[a, b] \rightarrow \mathbb{R}_{\mathcal{I}}^{+}$are two interval-valued $\gamma$-approximately h-convex functions, then

$$
\begin{aligned}
\frac{1}{h^{2}\left(\frac{1}{2}\right)} & F\left(\frac{a+b}{2}\right) G\left(\frac{a+b}{2}\right) \\
& -\frac{\epsilon}{h\left(\frac{1}{2}\right) \psi(1)}\left[(I R) \int_{\frac{a+b}{2}}^{b} \frac{\varphi(b-x)}{b-x}[F(x)+G(x)](\|2 x-(a+b)\|)^{\gamma} d x\right. \\
& \left.+(I R) \int_{a}^{\frac{a+b}{2}} \frac{\varphi(x-a)}{x-a}[F(x)+G(x)](\|a+b-2 x\|)^{\gamma} d x\right]
\end{aligned}
$$




$$
\begin{aligned}
\supseteq & \frac{1}{\psi(1)}\left[\left(\frac{a+b}{2}\right)^{+} \Im_{\varphi} F(b) G(b)+{ }_{\left(\frac{a+b}{2}\right)^{-}} \Im_{\varphi} F(a) G(a)\right] \\
& +\frac{1}{\psi(1) h^{2}\left(\frac{1}{2}\right)} \int_{\frac{a+b}{2}}^{b} \frac{\varphi(b-x)}{b-x} \epsilon^{2}(\|2 x-(a+b)\|)^{2 \gamma} d x \\
& +\frac{1}{\psi(1)}\left[K_{5} M(a, b)+K_{4} N(a, b)+\epsilon(\|b-a\|)^{\gamma} K_{6} P(a, b)\right]+2 \epsilon^{2}(\|b-a\|)^{2 \gamma} .
\end{aligned}
$$

Corollary 30 If $F, G:[a, b] \rightarrow \mathbb{R}_{\mathcal{I}}^{+}$are two interval-valued $\epsilon$-approximately h-convex functions, then

$$
\begin{aligned}
\frac{1}{h^{2}\left(\frac{1}{2}\right)} & F\left(\frac{a+b}{2}\right) G\left(\frac{a+b}{2}\right) \\
& -\frac{\epsilon}{h\left(\frac{1}{2}\right) \psi(1)}\left[(I R) \int_{\frac{a+b}{2}}^{b} \frac{\varphi(b-x)}{b-x}[F(x)+G(x)](\|2 x-(a+b)\|) d x\right. \\
& \left.+(I R) \int_{a}^{\frac{a+b}{2}} \frac{\varphi(x-a)}{x-a}[F(x)+G(x)](\|a+b-2 x\|) d x\right] \\
\supseteq & \frac{1}{\psi(1)}\left[\left(\frac{a+b}{2}\right)^{+} \Im_{\varphi} F(b) G(b)+\left(\frac{a+b}{2}\right)^{-} \Im_{\varphi} F(a) G(a)\right] \\
& +\frac{1}{\psi(1) h^{2}\left(\frac{1}{2}\right)} \int_{a}^{b} \frac{\varphi(b-x)}{b-x} \epsilon^{2}(\|2 x-(a+b)\|)^{2} d x \\
& +\frac{1}{\psi(1)}\left[K_{5} M(a, b)+K_{4} N(a, b)+\epsilon(\|b-a\|) K_{6} P(a, b)\right]+2 \epsilon^{2}(\|b-a\|)^{2} .
\end{aligned}
$$

From Theorem 12, we have the following result.

Corollary 31 If $F:[a, b] \rightarrow \mathbb{R}_{\mathcal{I}}^{+}$is interval-valued $\gamma$-approximately h-convex function, then

$$
\begin{aligned}
& \frac{1}{2 h\left(\frac{1}{2}\right)} F\left(\frac{a+b}{2}\right)-\frac{\epsilon}{2 \psi(1) h\left(\frac{1}{2}\right)} \int_{\frac{a+b}{2}}^{b} \frac{\varphi\left(x-\frac{a+b}{2}\right)}{x-\frac{a+b}{2}}(\|2 x-(a+b)\|)^{\gamma} d x \\
& \supseteq \frac{1}{2 \psi(1)}\left[{ }_{a+} \Im_{\varphi} F\left(\frac{a+b}{2}\right)+{ }_{b-} \Im_{\varphi} F\left(\frac{a+b}{2}\right)\right] \\
& \supseteq \frac{[F(a)+F(b)]}{2 \psi(1)} \int_{0}^{1} \frac{\varphi\left(\frac{(b-a)}{2} t\right)}{t}\left[h\left(\frac{1+t}{2}\right)+h\left(\frac{1-t}{2}\right)\right] d t+\epsilon(\|b-a\|)^{\gamma} .
\end{aligned}
$$

Corollary 32 If $F:[a, b] \rightarrow \mathbb{R}_{\mathcal{I}}^{+}$is interval-valued $\epsilon$-approximately h-convex function, then

$$
\begin{aligned}
& \frac{1}{2 h\left(\frac{1}{2}\right)} F\left(\frac{a+b}{2}\right)-\frac{\epsilon}{2 \psi(1) h\left(\frac{1}{2}\right)} \int_{\frac{a+b}{2}}^{b} \frac{\varphi\left(x-\frac{a+b}{2}\right)}{x-\frac{a+b}{2}}(\|2 x-(a+b)\|) d x \\
& \supseteq \supseteq \frac{1}{2 \psi(1)}\left[{ }_{a+} \Im_{\varphi} F\left(\frac{a+b}{2}\right)+{ }_{b-} \Im_{\varphi} F\left(\frac{a+b}{2}\right)\right] \\
& \supseteq \frac{[F(a)+F(b)]}{2 \psi(1)} \int_{0}^{1} \frac{\varphi\left(\frac{(b-a)}{2} t\right)}{t}\left[h\left(\frac{1+t}{2}\right)+h\left(\frac{1-t}{2}\right)\right] d t+\epsilon(\|b-a\|) .
\end{aligned}
$$

From Theorem 13, we get the following result. 
Corollary 33 If $F, G:[a, b] \rightarrow \mathbb{R}_{\mathcal{I}}^{+}$are two interval-valued $\gamma$-approximately h-convex functions, then

$$
\begin{aligned}
& {\left[a^{+} \Im_{\varphi} F\left(\frac{a+b}{2}\right) G\left(\frac{a+b}{2}\right)+b^{-} \Im_{\varphi} F\left(\frac{a+b}{2}\right) G\left(\frac{a+b}{2}\right)\right]} \\
& \supseteq K_{7} M(a, b)+K_{8} N(a, b)+\epsilon(\|b-a\|)^{\gamma} K_{9} P(a, b)+2 \psi(1) \epsilon^{2}(\|b-a\|)^{2 \gamma} .
\end{aligned}
$$

Corollary 34 If $F, G:[a, b] \rightarrow \mathbb{R}_{\mathcal{I}}^{+}$are two interval-valued $\epsilon$-approximately h-convex functions, then

$$
\begin{aligned}
& {\left[a^{+} \Im_{\varphi} F\left(\frac{a+b}{2}\right) G\left(\frac{a+b}{2}\right)+b^{-} \Im_{\varphi} F\left(\frac{a+b}{2}\right) G\left(\frac{a+b}{2}\right)\right]} \\
& \supseteq \supseteq K_{7} M(a, b)+K_{8} N(a, b)+\epsilon(\|b-a\|) K_{9} P(a, b)+2 \psi(1) \epsilon^{2}(\|b-a\|)^{2} .
\end{aligned}
$$

From Theorem 14, we obtain the following result.

Corollary 35 If $F, G:[a, b] \rightarrow \mathbb{R}_{\mathcal{I}}^{+}$are two interval-valued $\gamma$-approximately h-convex functions, then

$$
\begin{aligned}
\frac{1}{h^{2}\left(\frac{1}{2}\right)} F\left(\frac{a+b}{2}\right) G\left(\frac{a+b}{2}\right) & \\
& -\frac{\epsilon}{h\left(\frac{1}{2}\right) \psi(1)}\left[(I R) \int_{\frac{a+b}{2}}^{b} \frac{\varphi\left(x-\frac{a+b}{2}\right)}{x-\frac{a+b}{2}}[F(x)+G(x)](\|2 x-(a+b)\|)^{\gamma} d x\right. \\
& \left.+(I R) \int_{a}^{\frac{a+b}{2}} \frac{\varphi\left(\frac{a+b}{2}-x\right)}{\frac{a+b}{2}-x}[F(x)+G(x)](\|a+b-2 x\|)^{\gamma} d x\right] \\
\supseteq & \frac{1}{\psi(1)}\left[a^{+} \Im_{\varphi} F\left(\frac{a+b}{2}\right) G\left(\frac{a+b}{2}\right)+{ }_{b-} \Im_{\varphi} F\left(\frac{a+b}{2}\right) G\left(\frac{a+b}{2}\right)\right] \\
& +\frac{\epsilon^{2}}{\psi(1) h^{2}\left(\frac{1}{2}\right)} \int_{\frac{a+b}{2}}^{b} \frac{\varphi\left(x-\frac{a+b}{2}\right)}{x-\frac{a+b}{2}}(\|2 x-(a+b)\|)^{2 \gamma} d x \\
& +\frac{1}{\psi(1)}\left[K_{8} M(a, b)+K_{7} N(a, b)+\epsilon(\|b-a\|)^{\gamma} K_{9} P(a, b)\right]+2 \epsilon^{2}(\|b-a\|)^{2 \gamma} .
\end{aligned}
$$

Corollary 36 If $F, G:[a, b] \rightarrow \mathbb{R}_{\mathcal{I}}^{+}$are two interval-valued $\epsilon$-approximately h-convex functions, then

$$
\begin{aligned}
& \frac{1}{h^{2}\left(\frac{1}{2}\right)} F\left(\frac{a+b}{2}\right) G\left(\frac{a+b}{2}\right) \\
& \quad-\frac{\epsilon}{h\left(\frac{1}{2}\right) \psi(1)}\left[(I R) \int_{\frac{a+b}{2}}^{b} \frac{\varphi\left(x-\frac{a+b}{2}\right)}{x-\frac{a+b}{2}}[F(x)+G(x)](\|2 x-(a+b)\|) d x\right. \\
& \left.\quad+(I R) \int_{a}^{\frac{a+b}{2}} \frac{\varphi\left(\frac{a+b}{2}-x\right)}{\frac{a+b}{2}-x}[F(x)+G(x)](\|a+b-2 x\|) d x\right] \\
& \supseteq \frac{1}{\psi(1)}\left[a^{+} \Im_{\varphi} F\left(\frac{a+b}{2}\right) G\left(\frac{a+b}{2}\right)+{ }_{b} \Im_{\varphi} F\left(\frac{a+b}{2}\right) G\left(\frac{a+b}{2}\right)\right]
\end{aligned}
$$




$$
\begin{aligned}
& +\frac{\epsilon^{2}}{\psi(1) h^{2}\left(\frac{1}{2}\right)} \int_{\frac{a+b}{2}}^{b} \frac{\varphi\left(x-\frac{a+b}{2}\right)}{x-\frac{a+b}{2}}(\|2 x-(a+b)\|)^{2} d x \\
& +\frac{1}{\psi(1)}\left[K_{8} M(a, b)+K_{7} N(a, b)+\epsilon(\|b-a\|) K_{9} P(a, b)\right]+2 \epsilon^{2}(\|b-a\|)^{2} .
\end{aligned}
$$

Remark 4 Applying our Theorems 6-14, where $H(x, y)=-\mu t(1-t)\|y-x\|^{2}$ and $H(x, y)=$ $\mu t(1-t)\left\|\frac{1}{y}-\frac{1}{x}\right\|^{2}$ for some $\mu>0$, then we can obtain some new inequalities for intervalvalued strongly and relaxed $h$-convex functions via generalized fractional integrals.

Remark 5 Under the assumptions of Corollaries 2, 4, 6, if we take case V of Definition 3 then we have the results of Zhao et al. [1].

Remark 6 Under the assumptions of Corollaries 3, 5, 7, if we use case V of Definition 3 and $h(t)=t$, then we have the results of Budak et al. [2].

Remark 7 By using our Theorems 6-14, for suitable options of the function $\varphi(t)=\frac{t^{\frac{\alpha}{k}}}{k \Gamma_{k}(\alpha)}$; $\varphi(t)=t(x-t)^{\alpha-1}$ for $\alpha \in(0,1) ; \varphi(t)=\frac{t}{\alpha} \exp (-A t)$, where $A=\frac{1-\alpha}{\alpha}$ for $\alpha \in(0,1)$, etc., we can construct some new inequalities for interval-valued approximately $h$-convex functions. Finally, for appropriate choices of the function $h(t)=1 ; h(t)=t ; h(t)=t^{s} ; h(t)=t^{-s} ; h(t)=$ $t(1-t) ; h(t)=\frac{\sqrt{t}}{2 \sqrt{1-t}}$ etc., we can deduce some new interesting inequalities via generalized fractional integrals. We omit their proofs and the details are left to the interested reader.

\section{Conclusion}

This new class of functions called interval-valued approximately $h$-convex can be applied to obtain several new results in convex analysis, related optimization theory. The authors hope that this work may stimulate further research in different areas of pure and applied sciences.

\section{Acknowledgements}

The authors would like to express their sincere gratitude to the editor and the anonymous reviewers for their helpful comments and suggestions.

\section{Funding}

This work was supported in part by Special Soft Science Research Projects of Technological Innovation in Hubei Province (2019ADC146), the Fundamental Research Funds for Central Universities (2019B44914), Key Projects of Educational Commission of Hubei Province of China (D20192501), the Natural Science Foundation of Jiangsu Province (BK20180500), the National Key Research and Development Program of China (2018YFC1508100), and this project is partially supported by the National Natural Science Foundation of China (11971241).

Availability of data and materials

Not applicable.

\section{Competing interests}

The authors declare that they have no competing interests.

Authors' contributions

All authors contributed equally to the writing of this paper. All authors read and approved the final manuscript.

\section{Author details}

${ }^{1}$ College of Science, Hohai University, Nanjing, Jiangsu 210098, P.R. China. ${ }^{2}$ School of Mathematics and Statistics, Hubei Normal University, Huangshi, Hubei 435002, P.R. China. ${ }^{3}$ Jiangsu Key Laboratory for NSLSCS, School of Mathematical Sciences, Nanjing Normal University, Nanjing, Jiangsu 210023, China. ${ }^{4}$ Department of Mathematics, Faculty of Technical Science, University Ismail Qemali, 9400 Vlora, Albania. ${ }^{5}$ Department of Mathematics, Faculty of Science and Arts, Düzce University, Düzce, Turkey. 


\section{Publisher's Note}

Springer Nature remains neutral with regard to jurisdictional claims in published maps and institutional affiliations.

\section{Received: 26 December 2019 Accepted: 11 September 2020 Published online: 18 September 2020}

\section{References}

1. Zhao, D., An, T., Ye, G., Liu, W.: New Jensen and Hermite-Hadamard type inequalities for h-convex interval-valued functions. J. Inequal. Appl. 2018(1), 302 (2018)

2. Budak, H., Tunç, T., Sarikaya, M.: Fractional Hermite-Hadamard-type inequalities for interval-valued functions. Proc. Am. Math. Soc. (2019)

3. Dragomir, S., Pearce, C.: Selected topics on Hermite-Hadamard inequalities and applications. RGMIA monographs, Victoria University. http://rgmia.vu.edu.au/monographs (2004)

4. Peajcariaac, J.E., Tong, Y.L.: Convex Functions, Partial Orderings, and Statistical Applications. Academic Press, Bostan (1992)

5. Chen, F:: A note on Hermite-Hadamard inequalities for products of convex functions. J. Appl. Math. (2013)

6. Dragomir, S.S.: Inequalities of Hermite-Hadamard type for h-convex functions on linear spaces. Proyecciones 34(4), 323-341 (2015)

7. Dragomir, S.S.: Two mappings in connection to Hadamard's inequalities. J. Math. Anal. Appl. 167(1), 49-56 (1992)

8. Dragomir, S., Pecaric, J., Persson, L.-E.: Some inequalities of Hadamard type. Soochow J. Math. 21(3), 335-341 (1995)

9. Pachpatte, B.: On some inequalities for convex functions. RGMIA Res. Rep. Collect. 6(1), 1-9 (2003)

10. Wang, J., Li, X., Zhu, C., et al.: Refinements of Hermite-Hadamard type inequalities involving fractional integrals. Bull. Belg. Math. Soc. Simon Stevin 20(4), 655-666 (2013)

11. Sarikaya, M.Z., Ertugral, F.: On the generalized Hermite-Hadamard inequalities. Ann. Univ. Craioval Math. Comput. Sci. Ser. (2017)

12. Sarikaya, M., Yildirim, H.: On generalization of the Riesz potential. Indian J. Math. Math. Sci. 3(2), 231-235 (2007)

13. Ertugral, F., Sarikaya, M.Z.: Simpson type integral inequalities for generalized fractional integral. Rev. R. Acad. Cienc. Exactas Fís. Nat., Ser. A Mat. 113(4), 3115-3124 (2019)

14. Tseng, K.-L., Hwang, S.-R.: New Hermite-Hadamard-type inequalities and their applications. Filomat 30(14), 3667-3680 (2016)

15. Moore, R.E.: Interval Analysis. Prentice Hall, Englewood Cliffs (1966)

16. Chalco-Cano, Y., Flores-Franulic, A., Román-Flores, H.: Ostrowski type inequalities for interval-valued functions using generalized Hukuhara derivative. Comput. Appl. Math. 31(3) (2012)

17. Chalco-Cano, Y., Lodwick, W.A., Condori-Equice, W.: Ostrowski type inequalities and applications in numerical integration for interval-valued functions. Soft Comput. 19(11), 3293-3300 (2015)

18. Román-Flores, H., Chalco-Cano, Y., Lodwick, W.: Some integral inequalities for interval-valued functions. Comput. Appl. Math. 37(2), 1306-1318 (2018)

19. Costa, T.: Jensen's inequality type integral for fuzzy-interval-valued functions. Fuzzy Sets Syst. 327, 31-47 (2017)

20. Costa, T., Román-Flores, H.: Some integral inequalities for fuzzy-interval-valued functions. Inf. Sci. 420, 110-125 (2017)

21. Flores-Franulič, A., Chalco-Cano, Y., Román-Flores, H.: An Ostrowski type inequality for interval-valued functions. In: 2013 Joint IFSA World Congress and NAFIPS Annual Meeting (IFSA/NAFIPS), pp. 1459-1462. IEEE (2013)

22. Román-Flores, H., Chalco-Cano, Y., Silva, G.N.: A note on Gronwall type inequality for interval-valued functions. In: 2013 Joint IFSA World Congress and NAFIPS Annual Meeting (IFSA/NAFIPS), pp. 1455-1458. IEEE (2013)

23. Sadowska, E.: Hadamard inequality and a refinement of Jensen inequality for set valued functions. Results Math. 32(3-4), 332-337 (1997)

24. Mitroi, F.-C., Nikodem, K., Wasowicz, S.: Hermite-Hadamard inequalities for convex set-valued functions. Demonstr. Math. 46(4), 655-662 (2013)

25. Nikodem, K., Sanchez, J.L., Sanchez, L.: Jensen and Hermite-Hadamard inequalities for strongly convex set-valued maps. Math. Æterna 4(8), 979-987 (2014)

26. Aubin, J.P., Cellina, A.: Differential Inclusions: Set-Valued Maps and Viability Theory. Springer, Berlin (2012)

27. Markov, S.: On the algebraic properties of convex bodies and some applications. J. Convex Anal. 7(1), 129-166 (2000)

28. Lupulescu, V.: Fractional calculus for interval-valued functions. Fuzzy Sets Syst. 265, 63-85 (2015)

29. Markov, S.: Calculus for interval functions of a real variable. Computing 22(4), 325-337 (1979)

30. Dinghas, A.: Zum minkowskischen integralbegriff abgeschlossener mengen. Math. Z. 66(1), 173-188 (1956)

31. Piatek, B.: On the Riemann integral of set-valued functions. Zesz. Nauk. Mat. Stosow./Politech. Saska (2012)

32. Piatek, B.: On the sincov functional equation. Demonstr. Math. 38(4), 875-882 (2005)

33. Moore, R.E., Kearfott, R.B., Cloud, M.J.: Introduction to Interval Analysis. SIAM, Philadelphia (2009)

34. Zhao, D., Ye, G., Liu, W., Torres, D.F.: Some inequalities for interval-valued functions on time scales. Soft Comput. 23(15), 6005-6015 (2019)

35. Breckner, W.W.: Continuity of generalized convex and generalized concave set-valued functions. Rev. Anal. Numér Théor. Approx. 22(1), 39-51 (1993)

36. Osuna-Gomez, R., Jimenez-Gamero, M.D., Chalco-Cano, Y., Rojas-Medar, M.A.: Hadamard and Jensen inequalities for s-convex fuzzy processes. In: Soft Methodology and Random Information Systems, pp. 645-652. Springer, Berlin (2004)

37. Liu, X., Ye, G., Zhao, D., Liu, W.: Fractional Hermite-Hadamard type inequalities for interval-valued functions. J. Inequal. Appl. 2019(1), $266(2019)$ 